

\title{
Equidistribution of values of linear forms on a cubic hypersurface
}

\author{
Sam Chow
}

\begin{abstract}
Let $C$ be a cubic form with integer coefficients in $n$ variables, and let $h$ be the $h$-invariant of $C$. Let $L_{1}, \ldots, L_{r}$ be linear forms with real coefficients such that, if $\boldsymbol{\alpha} \in \mathbb{R}^{r} \backslash\{\boldsymbol{0}\}$, then $\boldsymbol{\alpha} \cdot \boldsymbol{L}$ is not a rational form. Assume that $h>16+8 r$. Let $\tau \in \mathbb{R}^{r}$, and let $\eta$ be a positive real number. We prove an asymptotic formula for the weighted number of integer solutions $\boldsymbol{x} \in[-P, P]^{n}$ to the system $C(\boldsymbol{x})=0$, $|\boldsymbol{L}(\boldsymbol{x})-\boldsymbol{\tau}|<\eta$. If the coefficients of the linear forms are algebraically independent over the rationals, then we may replace the $h$-invariant condition with the hypothesis $n>16+9 r$ and show that the system has an integer solution. Finally, we show that the values of $\boldsymbol{L}$ at integer zeros of $C$ are equidistributed modulo 1 in $\mathbb{R}^{r}$, requiring only that $h>16$.
\end{abstract}

\section{Introduction}

Recently Sargent [2014] used ergodic methods to establish the equidistribution of values of real linear forms on a rational quadric, subject to modest conditions. His ideas stemmed from quantitative refinements [Dani and Margulis 1993; Eskin et al. 1998] of Margulis' proof [1989] of the Oppenheim conjecture. Such techniques do not readily apply to higher-degree hypersurfaces. Our purpose here is to use analytic methods to obtain similar results on a cubic hypersurface.

Our first theorem is stated in terms of the $h$-invariant of a nontrivial rational cubic form $C$ in $n$ variables, which is defined to be the least positive integer $h$ such that

$$
C(\boldsymbol{x})=A_{1}(\boldsymbol{x}) B_{1}(\boldsymbol{x})+\cdots+A_{h}(\boldsymbol{x}) B_{h}(\boldsymbol{x})
$$

identically, for some rational linear forms $A_{1}, \ldots, A_{h}$ and some rational quadratic forms $B_{1}, \ldots, B_{h}$. The $h$-invariant describes the geometry of the hypersurface $\{C=0\}$, and in fact $n-h$ is the greatest affine dimension of any rational linear space contained in this hypersurface (therefore $1 \leqslant h \leqslant n$ ).

MSC2010: primary 11D25; secondary 11D75, 11J13, 11J71, 11P55.

Keywords: diophantine equations, diophantine inequalities, diophantine approximation, equidistribution. 
Theorem 1.1. Let $C$ be a cubic form with integer coefficients in $n$ variables, and let $h=h(C)$ be the $h$-invariant of $C$. Let $L_{1}, \ldots, L_{r}$ be linear forms with real coefficients in $n$ variables such that, if $\boldsymbol{\alpha} \in \mathbb{R}^{r} \backslash\{\mathbf{0}\}$, then $\boldsymbol{\alpha} \cdot \boldsymbol{L}$ is not a rational form. Assume that

$$
h>16+8 r .
$$

Let $\boldsymbol{\tau} \in \mathbb{R}^{r}$ and $\eta>0$. Let

$$
w(\boldsymbol{x})=\left\{\begin{array}{cl}
\exp \left(-\sum_{j \leqslant n} 1 /\left(1-x_{j}^{2}\right)\right) & \text { if }|\boldsymbol{x}|<1, \\
0 & \text { if }|\boldsymbol{x}| \geqslant 1,
\end{array}\right.
$$

and define the weighted counting function

Then

$$
N_{w}(P)=\sum_{\substack{\boldsymbol{x} \in \mathbb{Z}^{n}: C(\boldsymbol{x})=0 \\ \text { and }|\boldsymbol{L}(\boldsymbol{x})-\boldsymbol{\tau}|<\eta}} w(\boldsymbol{x} / P) .
$$

$$
N_{w}(P)=(2 \eta)^{r} \mathfrak{S} \chi_{w} P^{n-r-3}+o\left(P^{n-r-3}\right)
$$

as $P \rightarrow \infty$, where

$$
\mathfrak{S}=\sum_{q \in \mathbb{N}} q^{-n} \sum_{\substack{a \bmod q \\(a, q)=1}} \sum_{x \bmod q} e_{q}(a C(\boldsymbol{x}))
$$

and

$$
\chi_{w}=\int_{\mathbb{R}^{r+1}} \int_{\mathbb{R}^{n}} w(\boldsymbol{x}) e\left(\beta_{0} C(\boldsymbol{x})+\boldsymbol{\alpha} \cdot \boldsymbol{L}(\boldsymbol{x})\right) \mathrm{d} \boldsymbol{x} \mathrm{d} \beta_{0} \mathrm{~d} \boldsymbol{\alpha} .
$$

Further, we have $\mathfrak{S} \chi_{w}>0$.

The condition that no form in the real pencil of the linear forms is rational cannot be avoided, for if $|\boldsymbol{L}(\boldsymbol{x})-\boldsymbol{\tau}|<\eta$, then $|\boldsymbol{\alpha} \cdot \boldsymbol{L}(\boldsymbol{x})-\boldsymbol{\alpha} \cdot \boldsymbol{\tau}|<\eta|\boldsymbol{\alpha}|$, and the values taken by a rational form at integer points are discrete. As a simple example, the inequality

$$
\left|x_{1}+\cdots+x_{n}-\frac{1}{2}\right|<\frac{1}{4}
$$

admits no integer solutions $\boldsymbol{x}$.

We interpret $N_{w}(P)$ as a weighted count for the number of integer solutions $\boldsymbol{x} \in(-P, P)^{n}$ to the system

$$
C(\boldsymbol{x})=0, \quad|\boldsymbol{L}(\boldsymbol{x})-\boldsymbol{\tau}|<\eta .
$$

The smooth weight function $w(\boldsymbol{x})$ defined in (1-3) is taken from [Heath-Brown 1996]. Importantly, it has bounded support and bounded partial derivatives of all orders. One advantage of the weighted approach is that it enables the use of Poisson summation.

The singular series $\mathfrak{S}$ may be interpreted as a product of $p$-adic densities of points on the hypersurface $\{C=0\}$ [Birch 1962, §7]. Note that this captures the 
arithmetic of $C$ but that no such arithmetic is present for the linear forms $L_{1}, \ldots, L_{r}$ since they are "irrational" in a precise sense.

The weighted singular integral $\chi_{w}$ arises naturally in our proof as the right-hand side of (1-6). Using [Schmidt 1982b; 1985], we can interpret $\chi_{w}$ as the weighted real density of points on the variety $\left\{C=L_{1}=\cdots=L_{r}=0\right\}$. For $L>0$ and $\xi \in \mathbb{R}$, let

$$
\psi_{L}(\xi)=L \cdot \max (0,1-L|\xi|) .
$$

For $\xi \in \mathbb{R}^{r+1}$, put

$$
\Psi_{L}(\xi)=\prod_{v \leqslant r+1} \psi_{L}\left(\xi_{v}\right)
$$

With $f=(C, L)$, set

and define

$$
I_{L}(\boldsymbol{f})=\int_{\mathbb{R}^{n}} w(\boldsymbol{x}) \Psi_{L}(\boldsymbol{f}(\boldsymbol{x})) \mathrm{d} \boldsymbol{x}
$$

$$
\chi_{w}=\lim _{L \rightarrow \infty} I_{L}(\boldsymbol{f})
$$

We shall see that the limit (1-8) exists and that this definition is equivalent to the analytic definition (1-6).

We may replace the condition on the $h$-invariant by a condition on the number of variables, at the expense of assuming that the coefficients of the linear forms are in "general position".

Theorem 1.2. Let $C$ be a cubic form with rational coefficients in $n$ variables. Let $L_{1}, \ldots, L_{r}$ be linear forms in $n$ variables with real coefficients that are algebraically independent over $\mathbb{Q}$. Assume that

$$
n>16+9 r .
$$

Let $\boldsymbol{\tau} \in \mathbb{R}^{r}$ and $\eta>0$. Then there exists $\boldsymbol{x} \in \mathbb{Z}^{n}$ satisfying (1-7).

This algebraic independence condition is stronger than the real pencil condition imposed on the linear forms in Theorem 1.1-we show in Section 8 that the algebraic independence condition in fact implies the real pencil condition. The real pencil condition is probably sufficient, in truth; however, our proof relies on algebraic independence.

The point of this work is to show that the zeros of a rational cubic form are, in a strong sense, well distributed. Similar methods may be applied if $C$ is replaced by a higher-degree form; the simplest results would concern nonsingular forms of odd degree. The unweighted analogue of the case $r=0$ of Theorem 1.1 has been solved, assuming only that $h \geqslant 16$; see remark (B) in the introduction of [Schmidt 1985]. For the case $r=0$ of Theorem 1.2, we can choose $\boldsymbol{x}=\mathbf{0}$ or note from [Heath-Brown 2007] that fourteen variables suffice to ensure a nontrivial solution. We shall assume throughout that $r \geqslant 1$. 
The fact that we have linear inequalities rather than equations does genuinely increase the difficulty of the problem. For example, suppose we wished to nontrivially solve the system of equations $C=L_{1}=\cdots=L_{r}=0$, where here the $L_{i}$ are linear forms with rational coefficients; assume for simplicity that the $L_{i}$ are linearly independent. Using the linear equations, we could determine $r$ of the variables in terms of the remaining $n-r$ variables, and substituting into $C(\boldsymbol{x})=0$ would yield a homogeneous cubic equation in $n-r$ variables. Thus, by [Heath-Brown 2007], we could solve the system given $n \geqslant 14+r$ variables.

We use the work of Browning, Dietmann, and Heath-Brown [Browning et al. 2015] as a benchmark for comparison. Those authors investigate simultaneous rational solutions to one cubic equation $C=0$ and one quadratic equation $Q=0$. They establish the smooth Hasse principle under the assumption that

$$
\min (h(C), \operatorname{rank}(Q)) \geqslant 37 .
$$

We expect to do somewhat better when considering one cubic equation and one linear inequality simultaneously, and we do. Substituting $r=1$ into (1-2), we see that we only require $h(C)>24$.

To prove Theorem 1.1, we use the Hardy-Littlewood method [Vaughan 1997] in unison with Freeman's variant [2002] of the Davenport-Heilbronn method [1946]. The central objects to study are the weighted exponential sums

$$
S\left(\alpha_{0}, \boldsymbol{\alpha}\right)=\sum_{\boldsymbol{x} \in \mathbb{Z}^{n}} w(\boldsymbol{x} / P) e\left(\alpha_{0} C(\boldsymbol{x})+\boldsymbol{\alpha} \cdot \boldsymbol{L}(\boldsymbol{x})\right) .
$$

If $\left|S\left(\alpha_{0}, \boldsymbol{\alpha}\right)\right|$ is substantially smaller than the trivial estimate $O\left(P^{n}\right)$, then we may adapt [Davenport and Lewis 1964, Lemma 4] to rationally approximate $\alpha_{0}$ (see Section 2).

In Section 3, we use Poisson summation to approximately decompose our exponential sum into archimedean and nonarchimedean components. In Section 4, we use Heath-Brown's first-derivative bound [1996, Lemma 10] and a classical pruning argument [Davenport 2005, Lemma 15.1] to essentially obtain good simultaneous rational approximations to $\alpha_{0}$ and $\boldsymbol{\alpha}$. In Section 5, we combine classical ideas with Heath-Brown's first-derivative bound to obtain a mean-value estimate of the correct order of magnitude. In Section 6, we define our Davenport-Heilbronn arcs and in particular use the methods of Bentkus, Götze, and Freeman [Bentkus and Götze 1999; Freeman 2002; Wooley 2003] to obtain nontrivial cancellation on the minor arcs, thereby establishing the asymptotic formula (1-4). We complete the proof of Theorem 1.1 in Section 7 by explaining why $\mathfrak{S}$ and $\chi_{w}$ are positive. It is then that we justify the interpretation of $\chi_{w}$ as a weighted real density.

We prove Theorem 1.2 in Section 8. By Theorem 1.1, it suffices to consider the case where the $h$-invariant is not too large. With $A_{1}, \ldots, A_{h}$ as in (1-1), we solve 
the system (1-7) by solving the linear system

$$
\boldsymbol{A}(\boldsymbol{x})=\mathbf{0}, \quad|\boldsymbol{L}(\boldsymbol{x})-\boldsymbol{\tau}|<\eta .
$$

Since the $h$-invariant is not too large, this system has more variables than constraints and can be solved using methods from linear algebra and diophantine approximation, provided that the coefficients of $L_{1}, \ldots, L_{r}$ are algebraically independent over $\mathbb{Q}$.

In Section 9, we shall prove the following equidistribution result.

Theorem 1.3. Let $C$ be a cubic form with rational coefficients in $n$ variables, let $h=h(C)$ be the $h$-invariant of $C$, and assume that $h>16$. Let $r \in \mathbb{N}$, and let $L_{1}, \ldots, L_{r}$ be linear forms with real coefficients in $n$ variables such that, if $\boldsymbol{\alpha} \in \mathbb{R}^{r} \backslash\{\mathbf{0}\}$, then $\boldsymbol{\alpha} \cdot \boldsymbol{L}$ is not a rational form. Let

$$
Z=\left\{\boldsymbol{x} \in \mathbb{Z}^{n}: C(\boldsymbol{x})=0\right\},
$$

and order this set by height $|\boldsymbol{x}|$. Then the values of $\boldsymbol{L}(Z)$ are equidistributed modulo 1 in $\mathbb{R}^{r}$.

A little surprisingly, perhaps, we do not require $h$ to grow with $r$. By a multidimensional Weyl criterion [Cassels 1957, p. 66], it will suffice to investigate $S_{u}\left(\alpha_{0}, \boldsymbol{k}\right)$ for a fixed nonzero integer vector $\boldsymbol{k}$, where

$$
S_{u}\left(\alpha_{0}, \boldsymbol{\alpha}\right)=\sum_{|\boldsymbol{x}|<P} e\left(\alpha_{0} C(\boldsymbol{x})+\boldsymbol{\alpha} \cdot \boldsymbol{L}(\boldsymbol{x})\right)
$$

is the unweighted analogue of $S\left(\alpha_{0}, \alpha\right)$. A simplification of the method employed to prove Theorem 1.1 will complete the argument.

Rather than using the $h$-invariant, one could instead consider the dimension of the singular locus of the affine variety $\{C=0\}$, as in [Birch 1962]. Such an analysis would imply results for arbitrary nonsingular cubic forms in sufficiently many variables. These types of theorems are discussed in Section 10.

We adopt the convention that $\varepsilon$ denotes an arbitrarily small positive number, so its value may differ between instances. For $x \in \mathbb{R}$ and $q \in \mathbb{N}$, we put $e(x)=e^{2 \pi i x}$ and $e_{q}(x)=e^{2 \pi i x / q}$. Boldface will be used for vectors; for instance we shall abbreviate $\left(x_{1}, \ldots, x_{n}\right)$ to $\boldsymbol{x}$ and define $|\boldsymbol{x}|=\max \left(\left|x_{1}\right|, \ldots,\left|x_{n}\right|\right)$. We will use the unnormalized sinc function, given by $\operatorname{sinc}(x)=\sin (x) / x$ for $x \in \mathbb{R} \backslash\{0\}$ and $\operatorname{sinc}(0)=1$. For $x \in \mathbb{R}$, we write $\|x\|$ for the distance from $x$ to the nearest integer.

We regard $\boldsymbol{\tau}$ and $\eta$ as constants. The word large shall mean in terms of $C, \boldsymbol{L}$, $\varepsilon$, and constants, together with any explicitly stated dependence. Similarly, the implicit constants in Vinogradov's and Landau's notation may depend on $C, \boldsymbol{L}, \varepsilon$, and constants, and any other dependence will be made explicit. The pronumeral $P$ denotes a large positive real number. The word small will mean in terms of $C$, $\boldsymbol{L}$, and constants. We sometimes use such language informally, for the sake of motivation; we make this distinction using quotation marks. 


\section{One rational approximation}

First we use Freeman's kernel functions $[2002, \S 2.1]$ to relate $N_{w}(P)$ to our exponential sums $S\left(\alpha_{0}, \boldsymbol{\alpha}\right)$. We shall define

$$
T:[1, \infty) \rightarrow[1, \infty)
$$

in due course. For now, it suffices to note that

$$
T(P) \leqslant P
$$

and that $T(P) \rightarrow \infty$ as $P \rightarrow \infty$. Put

$$
L(P)=\max (1, \log T(P)), \quad \rho=\eta L(P)^{-1},
$$

and

$$
K_{ \pm}(\alpha)=\frac{\sin (\pi \alpha \rho) \sin (\pi \alpha(2 \eta \pm \rho))}{\pi^{2} \alpha^{2} \rho} .
$$

From [Freeman 2002, Lemma 1] and its proof, we have

$$
K_{ \pm}(\alpha) \ll \min \left(1, L(P)|\alpha|^{-2}\right)
$$

and

$$
0 \leqslant \int_{\mathbb{R}} e(\alpha t) K_{-}(\alpha) \mathrm{d} \alpha \leqslant U_{\eta}(t) \leqslant \int_{\mathbb{R}} e(\alpha t) K_{+}(\alpha) \mathrm{d} \alpha \leqslant 1,
$$

where

$$
U_{\eta}(t)= \begin{cases}1 & \text { if }|t|<\eta \\ 0 & \text { if }|t| \geqslant \eta\end{cases}
$$

For $\boldsymbol{\alpha} \in \mathbb{R}^{r}$, write

$$
\mathbb{K}_{ \pm}(\boldsymbol{\alpha})=\prod_{k \leqslant r} K_{ \pm}\left(\alpha_{k}\right)
$$

Let $U$ be a unit interval, to be specified later. The inequalities (2-5) and the identity

$$
\int_{U} e(\alpha m) \mathrm{d} \alpha= \begin{cases}1 & \text { if } m=0 \\ 0 & \text { if } m \in \mathbb{Z} \backslash\{0\}\end{cases}
$$

now give

$$
R_{-}(P) \leqslant N_{w}(P) \leqslant R_{+}(P),
$$

where

$$
R_{ \pm}(P)=\int_{\mathbb{R}^{r}} \int_{U} S\left(\alpha_{0}, \boldsymbol{\alpha}\right) e(-\boldsymbol{\alpha} \cdot \boldsymbol{\tau}) \mathbb{K}_{ \pm}(\boldsymbol{\alpha}) \mathrm{d} \alpha_{0} \mathrm{~d} \boldsymbol{\alpha} .
$$

In order to prove (1-4), it therefore remains to show that

$$
R_{ \pm}(P)=(2 \eta)^{r} \mathfrak{S} \chi_{w} P^{n-r-3}+o\left(P^{n-r-3}\right) .
$$


We shall in fact need to investigate the more general exponential sum

$$
g\left(\alpha_{0}, \lambda\right)=\sum_{\boldsymbol{x} \in \mathbb{Z}^{n}} w(\boldsymbol{x} / P) e\left(\alpha_{0} C(\boldsymbol{x})+\boldsymbol{\lambda} \cdot \boldsymbol{x}\right) .
$$

We note at once that

$$
S\left(\alpha_{0}, \boldsymbol{\alpha}\right)=g\left(\alpha_{0}, \Lambda \boldsymbol{\alpha}\right)
$$

where

$$
L_{i}(\boldsymbol{x})=\lambda_{i, 1} x_{1}+\cdots+\lambda_{i, n} x_{n} \quad(1 \leqslant i \leqslant r)
$$

and

$$
\Lambda=\left(\begin{array}{ccc}
\lambda_{1,1} & \cdots & \lambda_{r, 1} \\
\vdots & & \vdots \\
\lambda_{1, n} & \cdots & \lambda_{r, n}
\end{array}\right)
$$

Fix a large positive constant $C_{1}$.

Lemma 2.1. If $0<\theta<1$ and

$$
\left|g\left(\alpha_{0}, \lambda\right)\right| \geqslant P^{n-(h / 4) \theta+\varepsilon},
$$

then there exist relatively prime integers $q$ and a satisfying

$$
1 \leqslant q \leqslant C_{1} P^{2 \theta}, \quad\left|q \alpha_{0}-a\right|<P^{2 \theta-3} .
$$

The same is true if we replace $g\left(\alpha_{0}, \lambda\right)$ by

$$
g_{u}\left(\alpha_{0}, \lambda\right):=\sum_{|\boldsymbol{x}|<P} e\left(\alpha_{0} C(\boldsymbol{x})+\lambda \cdot \boldsymbol{x}\right)
$$

or by

$$
\sum_{1 \leqslant x_{1}, \ldots, x_{n} \leqslant P} e\left(\alpha_{0} C(\boldsymbol{x})+\boldsymbol{\lambda} \cdot \boldsymbol{x}\right) .
$$

Proof. Our existence statement is a weighted analogue of [Davenport and Lewis 1964, Lemma 4]. One can follow [loc. cit., §3], mutatis mutandis. The only change required is in proving the analogue of [loc. cit., Lemma 1]. Weights are introduced into the linear exponential sums that arise from Weyl differencing, but these weights are easily handled using partial summation. Our final statement holds with the same proof: one imitates [loc. cit., §3].

For $\theta \in(0,1), q \in \mathbb{N}$, and $a \in \mathbb{Z}$, let $\mathfrak{N}_{q, a}(\theta)$ be the set of $\left(\alpha_{0}, \boldsymbol{\alpha}\right) \in U \times \mathbb{R}^{r}$ satisfying (2-11), and let $\mathfrak{N}(\theta)$ be the union of the sets $\mathfrak{N}_{q, a}(\theta)$ over relatively prime $q$ and $a$. This union is disjoint if $\theta<\frac{3}{4}$. Indeed, suppose we have (2-11) for some relatively prime integers $q$ and $a$ and that we also have relatively prime integers $q^{\prime}$ and $a^{\prime}$ satisfying

$$
1 \leqslant q^{\prime} \ll P^{2 \theta}, \quad\left|q^{\prime} \alpha_{0}-a^{\prime}\right|<P^{2 \theta-3} .
$$


The triangle inequality then yields

$$
\left|a / q-a^{\prime} / q^{\prime}\right|<P^{2 \theta-3}\left(1 / q+1 / q^{\prime}\right)<1 /\left(q q^{\prime}\right)
$$

as $P$ is large. Hence, $a / q=a^{\prime} / q^{\prime}$, so $a^{\prime}=a$ and $q^{\prime}=q$.

We prune our arcs using the well known procedure in [Davenport 2005, Lemma 15.1]. Fix a small positive real number $\delta$. The following corollary shows that we may restrict attention to $\mathfrak{N}\left(\frac{1}{2}-\delta\right)$.

Corollary 2.2. We have

$$
\int_{U \times \mathbb{R}^{r} \backslash \mathfrak{N}(1 / 2-\delta)}\left|S\left(\alpha_{0}, \boldsymbol{\alpha}\right) \mathbb{K}_{ \pm}(\boldsymbol{\alpha})\right| \mathrm{d} \alpha_{0} \mathrm{~d} \boldsymbol{\alpha}=o\left(P^{n-r-3}\right) .
$$

Proof. Choose real numbers $\psi_{1}, \ldots, \psi_{t-1}$ such that

$$
\frac{1}{2}-\delta=\psi_{0}<\psi_{1}<\cdots<\psi_{t-1}<\psi_{t}=0.8
$$

Dirichlet's approximation theorem [Vaughan 1997, Lemma 2.1] implies $\mathfrak{N}\left(\psi_{t}\right)=$ $U \times \mathbb{R}^{r}$. Let $\mathfrak{U}$ be an arbitrary unit hypercube in $r$ dimensions, and put $\mathcal{U}=U \times \mathfrak{U}$. Since

$$
\operatorname{meas}(\mathfrak{N}(\theta) \cap \mathcal{U}) \ll P^{4 \theta-3},
$$

Lemma 2.1 gives

$$
\int_{\left(\mathfrak{N}\left(\psi_{g}\right) \backslash \mathfrak{N}\left(\psi_{g-1}\right)\right) \cap \mathcal{U}}\left|S\left(\alpha_{0}, \boldsymbol{\alpha}\right)\right| \mathrm{d} \alpha_{0} \mathrm{~d} \boldsymbol{\alpha} \ll P^{4 \psi_{g}-3+n-h \psi_{g-1} / 4+\varepsilon} \quad(1 \leqslant g \leqslant t) .
$$

This is $O\left(P^{n-r-3-\varepsilon}\right)$ if $\psi_{g-1} / \psi_{g} \simeq 1$ since $\psi_{g-1} \geqslant \frac{1}{2}-\delta$ and $h \geqslant 17+8 r$. Thus, we can choose $\psi_{1}, \ldots, \psi_{t-1}$ with $t \ll 1$ satisfactorily to ensure that

$$
\int_{\mathcal{U} \backslash \mathfrak{N}(1 / 2-\delta)}\left|S\left(\alpha_{0}, \boldsymbol{\alpha}\right)\right| \mathrm{d} \alpha_{0} \mathrm{~d} \boldsymbol{\alpha} \ll P^{n-r-3-\varepsilon} .
$$

The desired inequality now follows from (2-1), (2-2), (2-4), and (2-6).

Thus, to prove (2-8) and hence (1-4), it remains to show that

$\int_{\mathfrak{N}\left(\frac{1}{2}-\delta\right)} S\left(\alpha_{0}, \boldsymbol{\alpha}\right) e(-\boldsymbol{\alpha} \cdot \boldsymbol{\tau}) \mathbb{K}_{ \pm}(\boldsymbol{\alpha}) \mathrm{d} \alpha_{0} \mathrm{~d} \boldsymbol{\alpha}=(2 \eta)^{r} \mathfrak{S} \chi_{w} P^{n-r-3}+o\left(P^{n-r-3}\right)$.

\section{Poisson summation}

Put

$$
\alpha_{0}=\frac{a}{q}+\beta_{0}, \quad \lambda=q^{-1} \boldsymbol{a}+\boldsymbol{\beta},
$$

where $q \geqslant 1$ and $a$ are relatively prime integers and where $\boldsymbol{a} \in \mathbb{Z}^{n}$. By periodicity,

$$
g\left(\alpha_{0}, \lambda\right)=\sum_{\boldsymbol{y} \bmod q} e_{q}(a C(\boldsymbol{y})+\boldsymbol{a} \cdot \boldsymbol{y}) I_{\boldsymbol{y}}\left(q, \beta_{0}, \boldsymbol{\beta}\right),
$$


where

$$
I_{\boldsymbol{y}}\left(q, \beta_{0}, \boldsymbol{\beta}\right)=\sum_{\boldsymbol{x} \equiv \boldsymbol{y} \bmod q} w(\boldsymbol{x} / P) e\left(\beta_{0} C(\boldsymbol{x})+\boldsymbol{\beta} \cdot \boldsymbol{x}\right)
$$

Since

$$
I_{\boldsymbol{y}}\left(q, \beta_{0}, \boldsymbol{\beta}\right)=\sum_{\boldsymbol{z} \in \mathbb{Z}^{n}} w\left(\frac{\boldsymbol{y}+q \boldsymbol{z}}{P}\right) e\left(\beta_{0} C(\boldsymbol{y}+q \boldsymbol{z})+\boldsymbol{\beta} \cdot(\boldsymbol{y}+q \boldsymbol{z})\right),
$$

Poisson summation yields

$$
I_{\boldsymbol{y}}\left(q, \beta_{0}, \boldsymbol{\beta}\right)=\sum_{\boldsymbol{c} \in \mathbb{Z}^{n}} \int_{\mathbb{R}^{n}} w\left(\frac{\boldsymbol{y}+q \boldsymbol{z}}{P}\right) e\left(\beta_{0} C(\boldsymbol{y}+q \boldsymbol{z})+\boldsymbol{\beta} \cdot(\boldsymbol{y}+q \boldsymbol{z})-\boldsymbol{c} \cdot \boldsymbol{z}\right) \mathrm{d} \boldsymbol{z} .
$$

Changing variables now gives

$$
I_{\boldsymbol{y}}\left(q, \beta_{0}, \boldsymbol{\beta}\right)=(P / q)^{n} \sum_{\boldsymbol{c} \in \mathbb{Z}^{n}} e_{q}(\boldsymbol{c} \cdot \boldsymbol{y}) I\left(P^{3} \beta_{0}, P(\boldsymbol{\beta}-\boldsymbol{c} / q)\right),
$$

where

$$
I\left(\gamma_{0}, \boldsymbol{\gamma}\right)=\int_{\mathbb{R}^{n}} w(\boldsymbol{x}) e\left(\gamma_{0} C(\boldsymbol{x})+\boldsymbol{\gamma} \cdot \boldsymbol{x}\right) \mathrm{d} \boldsymbol{x} .
$$

Write

$$
S_{q, a, \boldsymbol{a}}=\sum_{\boldsymbol{y} \bmod q} e_{q}(a C(\boldsymbol{y})+\boldsymbol{a} \cdot \boldsymbol{y})
$$

and let

$$
g_{0}\left(\alpha_{0}, \lambda\right)=(P / q)^{n} S_{q, a, a} I\left(P^{3} \beta_{0}, P \boldsymbol{\beta}\right)
$$

be the $\boldsymbol{c}=\mathbf{0}$ contribution to $g\left(\alpha_{0}, \lambda\right)$. Then

$$
g\left(\alpha_{0}, \lambda\right)-g_{0}\left(\alpha_{0}, \lambda\right)=(P / q)^{n} \sum_{\boldsymbol{c} \neq \mathbf{0}} S_{q, a, \boldsymbol{a}+\boldsymbol{c}} I\left(P^{3} \beta_{0}, P(\boldsymbol{\beta}-\boldsymbol{c} / q)\right) .
$$

We shall bound the right-hand side from above, in the case where we have (2-11) and $|\boldsymbol{\beta}| \leqslant 1 /(2 q)$. For future reference, we note that specializing $(q, a, \boldsymbol{a})=(1,0, \mathbf{0})$ in (3-4) gives

$$
g\left(\alpha_{0}, \lambda\right)-P^{n} I\left(P^{3} \alpha_{0}, P \lambda\right)=P^{n} \sum_{\boldsymbol{c} \neq \mathbf{0}} I\left(P^{3} \alpha_{0}, P \lambda-P \boldsymbol{c}\right) .
$$

We bound $S_{q, a, a}$ by imitating [Davenport 2005, Lemma 15.3].

Lemma 3.1. Let $q \geqslant 1$ and a be relatively prime integers, and let $\psi>0$. Then

$$
S_{q, a, a} \ll_{\psi} q^{n-h / 8+\psi} .
$$

Proof. Suppose for a contradiction that $q$ is large in terms of $\psi$ and

$$
\left|S_{q, a, a}\right|>q^{n-h / 8+\psi} \text {. }
$$


Recall that

$$
S_{q, a, \boldsymbol{a}}=\sum_{1 \leqslant y_{1}, \ldots, y_{n} \leqslant q} e_{q}(a C(\boldsymbol{y})+\boldsymbol{a} \cdot \boldsymbol{y}) .
$$

We may assume without loss that $\psi<1$. By Lemma 2.1, with $P=q$ and $\theta=\frac{1}{2}-\psi / n$, there exist $s, b \in \mathbb{Z}$ such that

$$
1 \leqslant s<q, \quad|s a / q-b|<q^{-1} .
$$

Now $b / s=a / q$, which is impossible because $(a, q)=1$ and $1 \leqslant s<q$.

Let $\boldsymbol{c} \in \mathbb{Z}^{n} \backslash\{\boldsymbol{0}\}$, and suppose we have (2-11) for some $\theta \in\left(0, \frac{1}{2}-\delta\right]$ and some relatively prime $q, a \in \mathbb{Z}$. Define $a_{j}$ by rounding $q \lambda_{j}$ to the nearest integer, rounding down if $q \lambda_{j}$ is half of an odd integer $(1 \leqslant j \leqslant n)$. Since $|\boldsymbol{c}| / q \geqslant 1 / q \geqslant 2|\boldsymbol{\beta}|$,

$$
|P(\boldsymbol{\beta}-\boldsymbol{c} / q)| \gg P|\boldsymbol{c}| / q \gg P / q \gg P^{3+2 \delta}\left|\beta_{0}\right| \text {. }
$$

Now [Heath-Brown 1996, Lemma 10] gives

$$
I\left(P^{3} \beta_{0}, P(\boldsymbol{\beta}-\boldsymbol{c} / q)\right) \ll(P|\boldsymbol{c}| / q)^{-n-\varepsilon} .
$$

By (3-4), (3-6), and (3-7),

$$
g\left(\alpha_{0}, \lambda\right)-g_{0}\left(\alpha_{0}, \lambda\right) \ll P^{-\varepsilon} q^{n+2 \varepsilon-h / 8} \ll q^{n-h / 8+\varepsilon} .
$$

Let $\mathfrak{U}$ be an arbitrary unit hypercube in $r$ dimensions, and put $\mathcal{U}=U \times \mathfrak{U}$. With $\theta=\frac{1}{2}-\delta$, we now have

$$
\int_{\mathfrak{N}(\theta) \cap \mathcal{U}}\left|S\left(\alpha_{0}, \boldsymbol{\alpha}\right)-S_{0}\left(\alpha_{0}, \boldsymbol{\alpha}\right)\right| \mathrm{d} \alpha_{0} \mathrm{~d} \boldsymbol{\alpha} \ll \sum_{q \leqslant C_{1} P^{2 \theta}} q^{n-h / 8+\varepsilon} P^{2 \theta-3},
$$

where for $\left(\alpha_{0}, \boldsymbol{\alpha}\right) \in \mathfrak{N}_{q, a}(\theta)$ with $(a, q)=1$ we have written

$$
S_{0}\left(\alpha_{0}, \boldsymbol{\alpha}\right)=g_{0}\left(\alpha_{0}, \Lambda \boldsymbol{\alpha}\right) .
$$

Hence,

$$
\int_{\mathfrak{N}(\theta) \cap \mathcal{U}}\left|S\left(\alpha_{0}, \boldsymbol{\alpha}\right)-S_{0}\left(\alpha_{0}, \boldsymbol{\alpha}\right)\right| \mathrm{d} \alpha_{0} \mathrm{~d} \boldsymbol{\alpha} \ll P^{4 \theta-3}\left(P^{2 \theta}\right)^{n-h / 8+\varepsilon} \ll P^{n-r-3-\varepsilon}
$$

since $h \geqslant 17+8 r$. The bounds (2-1), (2-2), (2-4), and (2-6) now yield

$$
\int_{\mathfrak{N}(\theta)}\left|S\left(\alpha_{0}, \boldsymbol{\alpha}\right)-S_{0}\left(\alpha_{0}, \boldsymbol{\alpha}\right)\right| \cdot\left|\mathbb{K}_{ \pm}(\boldsymbol{\alpha})\right| \mathrm{d} \alpha_{0} \mathrm{~d} \boldsymbol{\alpha}=o\left(P^{n-r-3}\right) .
$$

Thus, to prove (2-13) and hence (1-4), it suffices to show that

$$
\int_{\mathfrak{N}\left(\frac{1}{2}-\delta\right)} S_{0}\left(\alpha_{0}, \boldsymbol{\alpha}\right) e(-\boldsymbol{\alpha} \cdot \boldsymbol{\tau}) \mathbb{K}_{ \pm}(\boldsymbol{\alpha}) \mathrm{d} \alpha_{0} \mathrm{~d} \boldsymbol{\alpha}=(2 \eta)^{r} \mathfrak{S} \chi_{w} P^{n-r-3}+o\left(P^{n-r-3}\right) .
$$




\section{More rational approximations}

For $\theta \in\left(0, \frac{1}{2}\right]$ and integers $q, a, a_{1}, \ldots, a_{n}$, let $\mathfrak{R}_{q, a, \boldsymbol{a}}(\theta)$ denote the set of $\left(\alpha_{0}, \boldsymbol{\alpha}\right) \in$ $U \times \mathbb{R}^{r}$ satisfying

$$
\left|q \alpha_{0}-a\right|<P^{2 \theta-3}, \quad|q \Lambda \boldsymbol{\alpha}-\boldsymbol{a}|<P^{(2+\delta) \theta-1},
$$

and let $\mathfrak{R}(\theta)$ be the union of the sets $\mathfrak{R}_{q, a, a}(\theta)$ over integers $q, a, a_{1}, \ldots, a_{n}$ satisfying

$$
1 \leqslant q \leqslant C_{1} P^{2 \theta}, \quad(a, q)=1 .
$$

Note that this union is disjoint if $\theta<(2+\delta)^{-1}$. Let $\mathfrak{U}$ be an arbitrary unit hypercube in $r$ dimensions, and put $\mathcal{U}=U \times \mathfrak{U}$. Then

$$
\operatorname{meas}(\mathfrak{R}(\theta) \cap \mathcal{U}) \ll P^{4 \theta-3-r+(2+\delta) \theta r}
$$

since our hypothesis on $\boldsymbol{L}$ implies that $\Lambda$ has $r$ linearly independent rows.

Fix a small positive real number $\theta_{0}$. The following lemma shows that we may restrict attention to $\mathfrak{R}\left(\theta_{0}\right)$.

Lemma 4.1. We have

$$
\int_{\mathfrak{N}(1 / 2-\delta) \backslash \mathfrak{R}\left(\theta_{0}\right)}\left|S_{0}\left(\alpha_{0}, \boldsymbol{\alpha}\right) \mathbb{K}_{ \pm}(\boldsymbol{\alpha})\right| \mathrm{d} \alpha_{0} \mathrm{~d} \boldsymbol{\alpha}=o\left(P^{n-r-3}\right) .
$$

Proof. Note that $\mathfrak{N}\left(\frac{1}{2}-\delta\right) \subseteq \mathfrak{N}\left(\frac{1}{2}\right)=\mathfrak{R}\left(\frac{1}{2}\right)$. Let

$$
\left(\alpha_{0}, \boldsymbol{\alpha}\right) \in \mathfrak{N}\left(\frac{1}{2}-\delta\right) \cap \mathfrak{R}\left(\theta_{g}\right) \backslash \mathfrak{R}\left(\theta_{g-1}\right)
$$

for some $g \in\{1,2, \ldots, t\}$, where

$$
0<\theta_{0}<\theta_{1}<\cdots<\theta_{t}=\frac{1}{2} .
$$

First suppose that $\left|S\left(\alpha_{0}, \boldsymbol{\alpha}\right)\right| \geqslant P^{n-h \theta_{g-1} / 4+\varepsilon}$. By Lemma 2.1, there exist relatively prime integers $q$ and $a$ satisfying

$$
1 \leqslant q \leqslant C_{1} P^{2 \theta_{g-1}}, \quad\left|q \alpha_{0}-a\right|<P^{2 \theta_{g-1}-3} .
$$

Let $\beta_{0}, \boldsymbol{a}$, and $\boldsymbol{\beta}$ be as in Section 3, with $\boldsymbol{\lambda}=\Lambda \boldsymbol{\alpha}$. Since $\left(\alpha_{0}, \boldsymbol{\alpha}\right) \notin \mathfrak{R}\left(\theta_{g-1}\right)$, we must have $q|\boldsymbol{\beta}| \geqslant P^{(2+\delta) \theta_{g-1}-1}$. Now

$$
P|\boldsymbol{\beta}| \gg q^{-1} P^{(2+\delta) \theta_{g-1}} \gg P^{\delta \theta_{g-1}} P^{3}\left|\beta_{0}\right|,
$$

so [Heath-Brown 1996, Lemma 10] yields

$$
I\left(P^{3} \beta_{0}, P \boldsymbol{\beta}\right) \ll_{N}\left(q^{-1} P^{(2+\delta) \theta_{g-1}}\right)^{-N} \ll P^{-N \delta \theta_{g-1}}
$$

for any $N>0$. Choosing $N$ large now gives $S_{0}\left(\alpha_{0}, \boldsymbol{\alpha}\right) \ll 1$. 
Now suppose instead that $\left|S\left(\alpha_{0}, \boldsymbol{\alpha}\right)\right|<P^{n-h \theta_{g-1} / 4+\varepsilon}$. As $\left(\alpha_{0}, \boldsymbol{\alpha}\right) \in \mathfrak{N}\left(\frac{1}{2}-\delta\right)$, there exist relatively prime integers $q$ and $a$ such that $\left(\alpha_{0}, \boldsymbol{\alpha}\right) \in \mathfrak{N}_{q, a}\left(\frac{1}{2}-\delta\right)$. From (3-8),

$$
S\left(\alpha_{0}, \boldsymbol{\alpha}\right)-S_{0}\left(\alpha_{0}, \boldsymbol{\alpha}\right) \ll q^{n-h / 8+\varepsilon} \ll P^{n-h / 8},
$$

and now the triangle inequality yields

$$
S_{0}\left(\alpha_{0}, \boldsymbol{\alpha}\right) \ll \max \left(P^{n-h \theta_{g-1} / 4+\varepsilon}, P^{n-h / 8}\right)=P^{n-h \theta_{g-1} / 4+\varepsilon} .
$$

The bound (4-3) is valid in both cases, so

$$
\int\left|S_{0}\left(\alpha_{0}, \boldsymbol{\alpha}\right)\right| \mathrm{d} \alpha_{0} \mathrm{~d} \boldsymbol{\alpha} \ll P^{4 \theta_{g}-3-r+(2+\delta) \theta_{g} r} P^{n-h \theta_{g-1} / 4+\varepsilon},
$$

where the integral is over $\mathfrak{N}\left(\frac{1}{2}-\delta\right) \cap \mathcal{U} \cap \mathfrak{R}\left(\theta_{g}\right) \backslash \mathfrak{R}\left(\theta_{g-1}\right)$. The right-hand side is $O\left(P^{n-r-3-\varepsilon}\right)$ if $\theta_{g} / \theta_{g-1} \simeq 1$ since $h \geqslant 17+8 r$. We can therefore choose $\theta_{1}, \ldots, \theta_{t-1}$ with $t \ll 1$ satisfactorily to ensure that

$$
\int_{\mathcal{U} \cap \mathfrak{N}(1 / 2-\delta) \backslash \Re\left(\theta_{0}\right)}\left|S_{0}\left(\alpha_{0}, \boldsymbol{\alpha}\right)\right| \mathrm{d} \alpha_{0} \mathrm{~d} \boldsymbol{\alpha} \ll P^{n-r-3-\varepsilon} .
$$

The desired inequality now follows from (2-1), (2-2), (2-4), and (2-6).

Thus, to prove (3-9) and hence (1-4), it suffices to show that

$$
\int_{\mathfrak{R}} S_{0}\left(\alpha_{0}, \boldsymbol{\alpha}\right) e(-\boldsymbol{\alpha} \cdot \boldsymbol{\tau}) \mathbb{K}_{ \pm}(\boldsymbol{\alpha}) \mathrm{d} \alpha_{0} \mathrm{~d} \boldsymbol{\alpha}=(2 \eta)^{r} \mathfrak{S} \chi_{w} P^{n-r-3}+o\left(P^{n-r-3}\right),
$$

where now and henceforth we write

$$
\mathfrak{R}=\mathfrak{R}_{P}=\mathfrak{R}\left(\theta_{0}\right), \quad \mathfrak{R}(q, a, \boldsymbol{a})=\mathfrak{R}_{q, a, \boldsymbol{a}}\left(\theta_{0}\right) .
$$

We now choose our unit interval

$$
U=\left(P^{2 \theta_{0}-3}, 1+P^{2 \theta_{0}-3}\right] .
$$

This choice ensures that, if the conditions (4-1) and (4-2) hold with $\theta=\theta_{0}$ for some $\left(\alpha_{0}, \boldsymbol{\alpha}\right) \in \mathbb{R} \times \mathbb{R}^{r}$, then $\alpha_{0} \in U$ if and only if $1 \leqslant a \leqslant q$. In particular, the set $\mathfrak{R}$ is the disjoint union of the sets $\Re(q, a, \boldsymbol{a})$ over integers $q, a, a_{1}, \ldots, a_{n}$ satisfying

$$
1 \leqslant a \leqslant q \leqslant C_{1} P^{2 \theta_{0}}, \quad(a, q)=1 .
$$

\section{A mean-value estimate}

We begin by bounding $I\left(\gamma_{0}, \boldsymbol{\gamma}\right)$. In light of (3-5), the first step is to bound $g\left(\alpha_{0}, \lambda\right)$.

Lemma 5.1. Let $\xi$ be a small positive real number. Let $\alpha_{0} \in \mathbb{R}$ and $\lambda \in \mathbb{R}^{n}$ with $\left|\alpha_{0}\right|<P^{-3 / 2}$. Then

$$
g\left(\alpha_{0}, \lambda\right) \ll P^{n+\xi}\left(P^{3}\left|\alpha_{0}\right|\right)^{-h / 8}
$$


and

$$
g_{u}\left(\alpha_{0}, \lambda\right) \ll P^{n+\xi}\left(P^{3}\left|\alpha_{0}\right|\right)^{-h / 8},
$$

where $g_{u}\left(\alpha_{0}, \lambda\right)$ is given by $(2-12)$.

Proof. This follows from the argument of the corollary to [Birch 1962, Lemma 4.3], using Lemma 2.1.

Lemma 5.2. We have

$$
I\left(\gamma_{0}, \boldsymbol{\gamma}\right) \ll \frac{1}{1+\left(\left|\gamma_{0}\right|+|\boldsymbol{\gamma}|\right)^{h / 8-\varepsilon}} .
$$

Proof. As $I\left(\gamma_{0}, \boldsymbol{\gamma}\right) \ll 1$, we may assume that $\left|\gamma_{0}\right|+|\boldsymbol{\gamma}|$ is large. From (3-5),

$$
I\left(\gamma_{0}, \boldsymbol{\gamma}\right)=P^{-n} g\left(\gamma_{0} / P^{3}, \boldsymbol{\gamma} / P\right)-\sum_{\boldsymbol{c} \neq \mathbf{0}} I\left(\gamma_{0}, \boldsymbol{\gamma}-P \boldsymbol{c}\right) .
$$

Since $I\left(\gamma_{0}, \gamma\right)$ is independent of $P$, we are free to choose $P=\left(\left|\gamma_{0}\right|+|\boldsymbol{\gamma}|\right)^{n}$. By Lemma 5.1 and [Heath-Brown 1996, Lemma 10], we now have

$$
I\left(\gamma_{0}, \boldsymbol{\gamma}\right) \ll P^{\varepsilon / n}\left|\gamma_{0}\right|^{-h / 8}=\frac{\left(\left|\gamma_{0}\right|+|\boldsymbol{\gamma}|\right)^{\varepsilon}}{\left|\gamma_{0}\right|^{h / 8}} .
$$

Let $C_{2}$ be a large positive constant. If $|\gamma| \geqslant C_{2}\left|\gamma_{0}\right|$, then [Heath-Brown 1996, Lemma 10] yields $I\left(\gamma_{0}, \boldsymbol{\gamma}\right) \ll_{N}|\boldsymbol{\gamma}|^{-N} \ll_{N}\left(\left|\gamma_{0}\right|+|\boldsymbol{\gamma}|\right)^{-N}$ for any $N>0$ while, if $|\gamma|<C_{2}\left|\gamma_{0}\right|$, then (5-2) gives

$$
I\left(\gamma_{0}, \boldsymbol{\gamma}\right) \ll\left(\left|\gamma_{0}\right|+|\boldsymbol{\gamma}|\right)^{\varepsilon-h / 8} .
$$

The latter bound is valid in either case. As $\left|\gamma_{0}\right|+|\boldsymbol{\gamma}|$ is large, our proof is complete.

We now have all of the necessary ingredients to obtain a mean-value estimate of the correct order of magnitude. Let $\mathfrak{U}$ be an arbitrary unit hypercube in $r$ dimensions, and put $\mathcal{U}=U \times \mathfrak{U}$. Let $V \subseteq\{1,2, \ldots, n\}$ index $r$ linearly independent rows of $\Lambda$. When $\left(\alpha_{0}, \boldsymbol{\alpha}\right) \in \mathfrak{R}(q, a, \boldsymbol{a})$ and $(a, q)=1$, write

$$
F\left(\alpha_{0}, \boldsymbol{\alpha}\right)=F\left(\alpha_{0}, \boldsymbol{\alpha} ; P\right)=\prod_{v \leqslant n}\left(q+P\left|q \lambda_{v}-a_{v}\right|\right)^{-1},
$$

where $\lambda=\Lambda \boldsymbol{\alpha}$. As $h / 8>r+2$, Lemmas 3.1 and 5.2 imply that $S_{0}\left(\alpha_{0}, \boldsymbol{\alpha}\right) F\left(\alpha_{0}, \boldsymbol{\alpha}\right)^{-\varepsilon}$

$$
\ll P^{n} q^{-r-2-\varepsilon}\left(1+P^{3}\left|\alpha_{0}-a / q\right|\right)^{-1-\varepsilon} \prod_{v \in V}\left(1+P\left|\lambda_{v}-a_{v} / q\right|\right)^{-1-\varepsilon} .
$$


For each $q$, we can choose from $O\left(q^{r+1}\right)$ values of $a$ and $a_{v}(v \in V)$ for which $\mathfrak{R}(q, a, \boldsymbol{a}) \cap \mathcal{U}$ is nonempty. Thus, an invertible change of variables gives

$$
\begin{aligned}
& \int_{\mathfrak{R} \mathcal{U}}\left|S_{0}\left(\alpha_{0}, \boldsymbol{\alpha}\right)\right| F\left(\alpha_{0}, \boldsymbol{\alpha}\right)^{-\varepsilon} \mathrm{d} \alpha_{0} \mathrm{~d} \boldsymbol{\alpha} \\
& \ll P^{n} \sum_{q \in \mathbb{N}} q^{-1-\varepsilon} \int_{\mathbb{R}}\left(1+P^{3}\left|\beta_{0}\right|\right)^{-1-\varepsilon} \mathrm{d} \beta_{0} \cdot \int_{\mathbb{R}^{r}} \prod_{j \leqslant r}\left(1+P\left|\alpha_{j}^{\prime}\right|\right)^{-1-\varepsilon} \mathrm{d} \boldsymbol{\alpha}^{\prime} \\
& \ll P^{n-r-3} .
\end{aligned}
$$

Positivity has permitted us to complete the summation and the integrals to infinity for an upper bound.

\section{The Davenport-Heilbronn method}

In this section, we specify our Davenport-Heilbronn dissection and complete the proof of (1-4). The bound (5-3) will suffice on the Davenport-Heilbronn major and trivial arcs, but on the minor arcs, we shall need to bound $F\left(\alpha_{0}, \boldsymbol{\alpha}\right)$ nontrivially. Using the methods of Bentkus, Götze, and Freeman, as exposited in [Wooley 2003, Lemmas 2.2 and 2.3], we will show that $F\left(\alpha_{0}, \boldsymbol{\alpha}\right)=o(1)$ in the case that $|\boldsymbol{\alpha}|$ is of "intermediate" size. The success of our endeavor depends crucially on our irrationality hypothesis for $\boldsymbol{L}$.

In order for the argument to work, we need to essentially replace $F$ with a function $\mathcal{F}$ defined on $\mathbb{R}^{r}$. For $\boldsymbol{\alpha} \in \mathbb{R}^{r}$, let $\mathcal{F}(\boldsymbol{\alpha} ; P)$ be the supremum of the quantity

$$
\prod_{v \leqslant n}\left(q+P\left|q \lambda_{v}-a_{v}\right|\right)^{-1}
$$

over $q \in \mathbb{N}$ and $\boldsymbol{a} \in \mathbb{Z}^{n}$, where $\lambda=\Lambda \boldsymbol{\alpha}$. Note that, if $\left(\alpha_{0}, \boldsymbol{\alpha}\right) \in \mathfrak{R}_{P}$, then

$$
F\left(\alpha_{0}, \boldsymbol{\alpha} ; P\right) \leqslant \mathcal{F}(\boldsymbol{\alpha} ; P) .
$$

Moreover, since $\Lambda$ has full rank, we have

$$
|\alpha| \ll|\Lambda \alpha| \ll|\alpha| .
$$

Lemma 6.1. Let $0<V \leqslant W$. Then

$$
\sup _{V \leqslant|\Lambda \boldsymbol{\alpha}| \leqslant W} \mathcal{F}(\boldsymbol{\alpha} ; P) \rightarrow 0 \quad(P \rightarrow \infty) .
$$

Proof. Suppose for a contradiction that (6-3) is false. Then there exist $\psi>0$ and

$$
\left(\boldsymbol{\alpha}^{(m)}, P_{m}, q_{m}, \boldsymbol{a}^{(m)}\right) \in \mathbb{R}^{r} \times[1, \infty) \times \mathbb{N} \times \mathbb{Z}^{n} \quad(m \in \mathbb{N})
$$

such that the sequence $\left(P_{m}\right)$ increases monotonically to infinity and such that, if $m \in \mathbb{N}$, then 


$$
\begin{gathered}
V \leqslant\left|\lambda^{(m)}\right| \leqslant W, \\
\prod_{v \leqslant n}\left(q_{m}+P_{m}\left|q_{m} \lambda_{v}^{(m)}-a_{v}^{(m)}\right|\right)<\psi^{-1},
\end{gathered}
$$

where $\lambda^{(m)}=\Lambda \boldsymbol{\alpha}^{(m)}(m \in \mathbb{N})$. Now $q_{m}<\psi^{-1} \ll 1$, so $\left|\boldsymbol{a}^{(m)}\right| \ll 1$. In particular, there are only finitely many possible choices for $\left(q_{m}, \boldsymbol{a}^{(m)}\right)$, so this pair must take a particular value infinitely often, say $(q, a)$.

From (6-4), we see that $q \lambda^{(m)}$ converges to $\boldsymbol{a}$ on a subsequence. The sequence $\left(\left|\boldsymbol{\alpha}^{(m)}\right|\right)_{m}$ is bounded, so by compactness, we know that $\boldsymbol{\alpha}^{(m)}$ converges to some vector $\boldsymbol{\alpha}$ on a subsubsequence. Therefore, $q \Lambda \boldsymbol{\alpha}=\boldsymbol{a}$ and in particular $\Lambda \boldsymbol{\alpha}$ is a rational vector, so $\boldsymbol{\alpha} \cdot \boldsymbol{L}$ is a rational form. Note that $\boldsymbol{\alpha} \neq \mathbf{0}$ since $\left|\boldsymbol{\alpha}^{(m)}\right| \gg 1$. This contradicts our hypothesis on $\boldsymbol{L}$, thereby establishing (6-3).

Corollary 6.2. Let $\theta$ be a small positive real number. Then there exists a function $T:[1, \infty) \rightarrow[1, \infty)$, increasing monotonically to infinity, such that $T(P) \leqslant P^{\theta}$ and

$$
\sup _{P^{\theta-1} \leqslant|\Lambda \boldsymbol{\alpha}| \leqslant T(P)} \mathcal{F}(\boldsymbol{\alpha} ; P) \leqslant T(P)^{-1} .
$$

Proof. Lemma 6.1 yields a sequence $\left(P_{m}\right)$ of large positive real numbers such that

$$
\sup _{1 / m \leqslant|\Lambda \boldsymbol{\alpha}| \leqslant m} \mathcal{F}\left(\boldsymbol{\alpha} ; P_{m}\right) \leqslant 1 / m \text {. }
$$

We may assume that this sequence is increasing and that $P_{m}^{\theta} \geqslant m(m \in \mathbb{N})$. Define $T(P)$ by $T(P)=1\left(1 \leqslant P \leqslant P_{1}\right)$ and $T(P)=m\left(P_{m} \leqslant P<P_{m+1}\right)$. Note that $T(P) \leqslant P^{\theta}$ and that $T(P)$ increases monotonically to infinity. Now

$$
\sup _{T(P)^{-1} \leqslant|\Lambda \boldsymbol{\alpha}| \leqslant T(P)} \mathcal{F}(\boldsymbol{\alpha} ; P) \leqslant T(P)^{-1},
$$

for if $P \geqslant P_{m}$, then $\mathcal{F}(\boldsymbol{\alpha} ; P) \leqslant \mathcal{F}\left(\boldsymbol{\alpha} ; P_{m}\right)$.

The inequality (6-5) plainly holds if $P \leqslant P_{1}$. Thus, it remains to show that, if $P$ is large and

$$
|\Lambda \boldsymbol{\alpha}|<T(P)^{-1}<\mathcal{F}(\boldsymbol{\alpha} ; P),
$$

then $|\Lambda \boldsymbol{\alpha}|<P^{\theta-1}$. Suppose we have (6-6), with $P$ large. Writing $\lambda=\Lambda \boldsymbol{\alpha}$, we have

$$
\prod_{v \leqslant n}\left(q+P\left|q \lambda_{v}-a_{v}\right|\right)<T(P)
$$

for some $q \in \mathbb{N}$ and some $\boldsymbol{a} \in \mathbb{Z}^{n}$. Now $q<T(P)^{1 / n}$ and

$$
|q \lambda-\boldsymbol{a}|<T(P) / P
$$

so the triangle inequality and (6-6) give

$$
|\boldsymbol{a}|<T(P) / P+T(P)^{1 / n-1}<1 .
$$


Therefore, $\boldsymbol{a}=\mathbf{0}$, and so

$$
|\Lambda \boldsymbol{\alpha}| \leqslant|q \lambda|<T(P) / P \leqslant P^{\theta-1},
$$

completing the proof.

Let $C_{3}$ be a large positive constant. Let $T(P)$ be as in Corollary 6.2 with $\theta=\delta^{2} \theta_{0}$. We define our Davenport-Heilbronn major arc by

$$
\mathfrak{M}=\left\{\left(\alpha_{0}, \boldsymbol{\alpha}\right) \in \mathbb{R} \times \mathbb{R}^{r}:|\boldsymbol{\alpha}|<C_{3} P^{\delta^{2} \theta_{0}-1}\right\},
$$

our minor arcs by

$$
\mathfrak{m}=\left\{\left(\alpha_{0}, \boldsymbol{\alpha}\right) \in \mathbb{R} \times \mathbb{R}^{r}: C_{3} P^{\delta^{2} \theta_{0}-1} \leqslant|\boldsymbol{\alpha}| \leqslant C_{3}^{-1} T(P)\right\},
$$

and our trivial arcs by

$$
\mathfrak{t}=\left\{\left(\alpha_{0}, \boldsymbol{\alpha}\right) \in \mathbb{R} \times \mathbb{R}^{r}:|\boldsymbol{\alpha}|>C_{3}^{-1} T(P)\right\} .
$$

It follows from (6-1), (6-2), and (6-5) that

$$
\sup _{\mathfrak{R} \cap \mathfrak{m}} F\left(\alpha_{0}, \boldsymbol{\alpha}\right) \leqslant T(P)^{-1} .
$$

Let $\mathfrak{U}$ be an arbitrary unit hypercube in $r$ dimensions, and put $\mathcal{U}=U \times \mathfrak{U}$. By (5-3) and (6-7),

$$
\int_{\mathfrak{R} \cap \mathfrak{m} \cap \mathcal{U}}\left|S_{0}\left(\alpha_{0}, \boldsymbol{\alpha}\right)\right| \mathrm{d} \alpha_{0} \mathrm{~d} \boldsymbol{\alpha} \ll T(P)^{-\varepsilon} P^{n-r-3} .
$$

Now (2-2), (2-4), and (2-6) yield

Note that

$$
\int_{\mathfrak{R} \cap \mathfrak{m}}\left|S_{0}\left(\alpha_{0}, \boldsymbol{\alpha}\right) \mathbb{K}_{ \pm}(\boldsymbol{\alpha})\right| \mathrm{d} \alpha_{0} \mathrm{~d} \boldsymbol{\alpha}=o\left(P^{n-r-3}\right) .
$$

$$
0<F\left(\alpha_{0}, \boldsymbol{\alpha}\right) \leqslant 1 .
$$

Together with (2-2), (2-4), (2-6), and (5-3), this gives

$$
\begin{aligned}
\int_{\mathfrak{R} \cap \mathfrak{t}}\left|S_{0}\left(\alpha_{0}, \boldsymbol{\alpha}\right) \mathbb{K}_{ \pm}(\boldsymbol{\alpha})\right| \mathrm{d} \alpha_{0} \mathrm{~d} \boldsymbol{\alpha} & \ll P^{n-r-3} L(P)^{r} \sum_{n=0}^{\infty}\left(C_{3}^{-1} T(P)+n\right)^{-2} \\
& \ll P^{n-r-3} L(P)^{r} T(P)^{-1}=o\left(P^{n-r-3}\right) .
\end{aligned}
$$

Coupling (6-8) with (6-10) yields

$$
\int_{\mathfrak{R} \backslash \mathfrak{M}}\left|S_{0}\left(\alpha_{0}, \boldsymbol{\alpha}\right) \mathbb{K}_{ \pm}(\boldsymbol{\alpha})\right| \mathrm{d} \alpha_{0} \mathrm{~d} \boldsymbol{\alpha}=o\left(P^{n-r-3}\right) .
$$

Recall that to show (1-4) it remains to establish (4-4). Defining

$$
S_{1}=\int_{\mathfrak{R} \cap \mathfrak{M}} S_{0}\left(\alpha_{0}, \boldsymbol{\alpha}\right) e(-\boldsymbol{\alpha} \cdot \boldsymbol{\tau}) \mathbb{K}_{ \pm}(\boldsymbol{\alpha}) \mathrm{d} \alpha_{0} \mathrm{~d} \boldsymbol{\alpha},
$$


it now suffices to prove that

$$
S_{1}=(2 \eta)^{r} \mathfrak{S} \chi_{w} P^{n-r-3}+o\left(P^{n-r-3}\right) .
$$

By (2-3),

$$
K_{ \pm}(\alpha)=(2 \eta \pm \rho) \cdot \operatorname{sinc}(\pi \alpha \rho) \cdot \operatorname{sinc}(\pi \alpha(2 \eta \pm \rho)) .
$$

Now (2-1), (2-2), and the Taylor expansion of sinc $(\cdot)$ yield

$$
K_{ \pm}(\alpha)=2 \eta+O\left(L(P)^{-1}\right) \quad\left(|\alpha|<P^{-1 / 2}\right) .
$$

Substituting this into (2-6) shows that, if $\left(\alpha_{0}, \boldsymbol{\alpha}\right) \in \mathfrak{M}$, then

$$
\mathbb{K}_{ \pm}(\boldsymbol{\alpha})=(2 \eta)^{r}+O\left(L(P)^{-1}\right) .
$$

Moreover, it follows from (5-3) and (6-9) that

$$
\int_{\mathfrak{R} \cap \mathfrak{M}}\left|S_{0}\left(\alpha_{0}, \boldsymbol{\alpha}\right)\right| \mathrm{d} \alpha_{0} \mathrm{~d} \boldsymbol{\alpha} \ll P^{n-r-3} .
$$

From (6-11) and (6-12), we infer that

$$
S_{1}=(2 \eta)^{r} \int_{\mathfrak{R} \cap \mathfrak{M}} S_{0}\left(\alpha_{0}, \boldsymbol{\alpha}\right) e(-\boldsymbol{\alpha} \cdot \boldsymbol{\tau}) \mathrm{d} \alpha_{0} \mathrm{~d} \boldsymbol{\alpha}+o\left(P^{n-r-3}\right) .
$$

Thus, to prove (1-4), it remains to show that

$$
S_{2}=\mathfrak{S} \chi_{w} P^{n-r-3}+o\left(P^{n-r-3}\right),
$$

where

$$
S_{2}=\int_{\mathfrak{R} \cap \mathfrak{M}} S_{0}\left(\alpha_{0}, \boldsymbol{\alpha}\right) e(-\boldsymbol{\alpha} \cdot \boldsymbol{\tau}) \mathrm{d} \alpha_{0} \mathrm{~d} \boldsymbol{\alpha} .
$$

For $q \in \mathbb{N}$ and $a \in\{1,2, \ldots, q\}$, let $X(q, a)$ be the set of $\left(\alpha_{0}, \boldsymbol{\alpha}\right) \in \mathbb{R} \times \mathbb{R}^{r}$ satisfying

$$
q \leqslant C_{1} P^{2 \theta_{0}}, \quad\left|q \alpha_{0}-a\right|<P^{2 \theta_{0}-3} .
$$

Lemma 6.3. Assume (4-6). Then $\mathfrak{R}(q, a, \mathbf{0}) \cap \mathfrak{M}=X(q, a) \cap \mathfrak{M}$.

Proof. As $\mathfrak{R}(q, a, \mathbf{0}) \subseteq X(q, a)$, we have $\mathfrak{R}(q, a, \mathbf{0}) \cap \mathfrak{M} \subseteq X(q, a) \cap \mathfrak{M}$. Next, suppose that $\left(\alpha_{0}, \boldsymbol{\alpha}\right) \in X(q, a) \cap \mathfrak{M}$. Then $|\boldsymbol{\alpha}|<C_{3} P^{\delta^{2} \theta_{0}-1}$, so

$$
|\Lambda \boldsymbol{\alpha}| \ll P^{\delta^{2} \theta_{0}-1} .
$$

Now $|q \Lambda \boldsymbol{\alpha}| \ll P^{\left(2+\delta^{2}\right) \theta_{0}-1}$, and in particular, we have (4-1) with $\theta=\theta_{0}$ and $\boldsymbol{a}=\mathbf{0}$. Thus, we have $\left(\alpha_{0}, \boldsymbol{\alpha}\right) \in \mathfrak{R}(q, a, \mathbf{0})$, and plainly $\left(\alpha_{0}, \boldsymbol{\alpha}\right) \in \mathfrak{M}$.

Note also that, if $\left(\alpha_{0}, \boldsymbol{\alpha}\right) \in \mathfrak{R} \cap \mathfrak{M}$, then $\left(\alpha_{0}, \boldsymbol{\alpha}\right) \in \mathfrak{R}(q, a, \mathbf{0})$ for some $q, a \in \mathbb{Z}$ satisfying (4-6). Indeed, if $\left(\alpha_{0}, \boldsymbol{\alpha}\right) \in \mathfrak{R}(q, a, \boldsymbol{a}) \cap \mathfrak{M}$ for some $q$, $a$, and $\boldsymbol{a}$ satisfying (4-6), then the triangle inequality implies that $\boldsymbol{a}=\mathbf{0}$. By Lemma 6.3, we conclude 
that $\mathfrak{R} \cap \mathfrak{M}$ is the disjoint union of the sets $X(q, a) \cap \mathfrak{M}$ over $q, a \in \mathbb{Z}$ satisfying (4-6). Put

$$
V(q)=\left[-q^{-1} P^{2 \theta_{0}-3}, q^{-1} P^{2 \theta_{0}-3}\right] \quad(q \in \mathbb{N})
$$

and

$$
W=\left[-C_{3} P^{\delta^{2} \theta_{0}-1}, C_{3} P^{\delta^{2} \theta_{0}-1}\right]^{r} .
$$

Now

$$
S_{2}=\sum_{q \leqslant C_{1} P^{2 \theta_{0}}} \sum_{\substack{a=1 \\(a, q)=1}}^{q} \int_{V(q) \times W} f_{q, a}\left(\beta_{0}, \boldsymbol{\alpha}\right) e(-\boldsymbol{\alpha} \cdot \boldsymbol{\tau}) \mathrm{d} \beta_{0} \mathrm{~d} \boldsymbol{\alpha}
$$

where

$$
f_{q, a}\left(\beta_{0}, \boldsymbol{\alpha}\right)=(P / q)^{n} S_{q, a, 0} I\left(P^{3} \beta_{0}, P \Lambda \boldsymbol{\alpha}\right) .
$$

To prove (6-13), we complete the integrals and the outer sum to infinity. In light of (1-2), it follows from Lemmas 3.1 and 5.2 that, if $(a, q)=1$, then

$$
f_{q, a}\left(\beta_{0}, \boldsymbol{\alpha}\right) \ll P^{n} q^{-3}\left(1+P^{3}\left|\beta_{0}\right|\right)^{-1-\varepsilon} \prod_{v \in V}\left(1+P\left|\lambda_{v}\right|\right)^{-1-\varepsilon},
$$

where $V$ is as in Section 5 and $\lambda=\Lambda \boldsymbol{\alpha}$. Let

$$
S_{3}=\sum_{q \leqslant C_{1} P^{2 \theta_{0}}} \sum_{\substack{a=1 \\(a, q)=1}}^{q} \int_{\mathbb{R} \times W} f_{q, a}\left(\beta_{0}, \boldsymbol{\alpha}\right) e(-\boldsymbol{\alpha} \cdot \boldsymbol{\tau}) \mathrm{d} \beta_{0} \mathrm{~d} \boldsymbol{\alpha} .
$$

By (6-15) and an invertible change of variables,

$$
\begin{aligned}
S_{2}-S_{3} & \ll P^{n} \sum_{q \in \mathbb{N}} q^{-3} \sum_{\substack{a=1 \\
(a, q)=1}}^{q} \int_{q^{-1} P^{2 \theta_{0}-3}}^{\infty}\left(P^{3} \beta_{0}\right)^{-1-\varepsilon} \mathrm{d} \beta_{0} \int_{\mathbb{R}^{r}} \prod_{v \in V}\left(1+P\left|\lambda_{v}\right|\right)^{-1-\varepsilon} \mathrm{d} \lambda_{V} \\
& =o\left(P^{n-r-3}\right),
\end{aligned}
$$

where $\lambda_{V}=\left(\lambda_{v}\right)_{v \in V}$.

Let

$$
S_{4}=\sum_{q \leqslant C_{1} P^{2 \theta_{0}}} \sum_{\substack{a=1 \\(a, q)=1}}^{q} \int_{\mathbb{R}^{r+1}} f_{q, a}\left(\beta_{0}, \boldsymbol{\alpha}\right) e(-\boldsymbol{\alpha} \cdot \boldsymbol{\tau}) \mathrm{d} \beta_{0} \mathrm{~d} \boldsymbol{\alpha} .
$$

By (6-15) and an invertible change of variables,

$$
S_{3}-S_{4} \ll P^{n} \sum_{q \in \mathbb{N}} q^{-3} \sum_{\substack{a=1 \\(a, q)=1}}^{q} \int_{\mathbb{R}}\left(1+P^{3}\left|\beta_{0}\right|\right)^{-1-\varepsilon} \mathrm{d} \beta_{0} \int \prod_{j \leqslant r}\left(1+P\left|\alpha_{j}^{\prime}\right|\right)^{-1-\varepsilon} \mathrm{d} \boldsymbol{\alpha}^{\prime} .
$$

Here the inner integral is over $\boldsymbol{\alpha}^{\prime} \in \mathbb{R}^{r}$ such that $\Lambda_{V}^{-1} \boldsymbol{\alpha}^{\prime} \notin W$, where $\Lambda_{V}$ is the 
submatrix of $\Lambda$ determined by taking rows indexed by $V$. With $c$ a small positive constant, we now have

$$
S_{3}-S_{4} \ll P^{n-r-2} \int_{c P^{\delta^{2} \theta_{0}-1}}^{\infty}(P \alpha)^{-1-\varepsilon} \mathrm{d} \alpha=o\left(P^{n-r-3}\right) .
$$

Let

$$
S_{5}=\sum_{q \in \mathbb{N}} \sum_{\substack{a=1 \\(a, q)=1}}^{q} \int_{\mathbb{R}^{r+1}} f_{q, a}\left(\beta_{0}, \boldsymbol{\alpha}\right) e(-\boldsymbol{\alpha} \cdot \boldsymbol{\tau}) \mathrm{d} \beta_{0} \mathrm{~d} \boldsymbol{\alpha} .
$$

By (6-15) and an invertible change of variables,

$$
\begin{aligned}
S_{4}-S_{5} & \ll P^{n} \sum_{q>C_{1} P^{2 \theta_{0}}} q^{-3} \sum_{\substack{a=1 \\
(a, q)=1}}^{q} \int_{\mathbb{R}}\left(1+P^{3}\left|\beta_{0}\right|\right)^{-1-\varepsilon} \mathrm{d} \beta_{0} \int_{\mathbb{R}^{r}} \prod_{j \leqslant r}\left(1+P\left|\alpha_{j}^{\prime}\right|\right)^{-1-\varepsilon} \mathrm{d} \boldsymbol{\alpha}^{\prime} \\
& \ll P^{n-r-3} \sum_{q>C_{1} P^{2 \theta_{0}}} q^{-2}=o\left(P^{n-r-3}\right) .
\end{aligned}
$$

In view of (1-5), (3-3), and (6-14),

$$
S_{5}=P^{n} \mathfrak{S} \int_{\mathbb{R}^{r+1}} e(-\boldsymbol{\alpha} \cdot \boldsymbol{\tau}) I\left(P^{3} \beta_{0}, P \Lambda \boldsymbol{\alpha}\right) \mathrm{d} \beta_{0} \mathrm{~d} \boldsymbol{\alpha} .
$$

Changing variables yields

$$
S_{5}=P^{n-r-3} \mathfrak{S} \int_{\mathbb{R}^{r+1}} e\left(-P^{-1} \boldsymbol{\alpha} \cdot \boldsymbol{\tau}\right) I\left(\beta_{0}, \Lambda \boldsymbol{\alpha}\right) \mathrm{d} \beta_{0} \mathrm{~d} \boldsymbol{\alpha} .
$$

By (1-6) and (3-2),

$$
\chi_{w}=\int_{\mathbb{R}^{r+1}} I\left(\beta_{0}, \Lambda \boldsymbol{\alpha}\right) \mathrm{d} \beta_{0} \mathrm{~d} \boldsymbol{\alpha} .
$$

As $h \geqslant 17+8 r$, the bounds (5-1) and

$$
e\left(-P^{-1} \boldsymbol{\alpha} \cdot \boldsymbol{\tau}\right)-1 \ll P^{-1}|\boldsymbol{\alpha}|
$$

imply that

$$
\int_{\mathbb{R}^{r+1}} e\left(-P^{-1} \boldsymbol{\alpha} \cdot \boldsymbol{\tau}\right) I\left(\beta_{0}, \Lambda \boldsymbol{\alpha}\right) \mathrm{d} \beta_{0} \mathrm{~d} \boldsymbol{\alpha}=\chi_{w}+O\left(P^{-1}\right)
$$

Substituting this into (6-19) yields

$$
S_{5}=P^{n-r-3} \mathfrak{S} \chi_{w}+o\left(P^{n-r-3}\right) .
$$

Combining this with (6-16), (6-17), and (6-18) yields (6-13), completing the proof of (1-4). 


\section{Positivity of the singular series and singular integral}

In this section, we confirm that $\mathfrak{S}>0$ and $\chi_{w}>0$, thereby completing the proof of Theorem 1.1. Since $\mathfrak{S}$ is the singular series associated to the cubic form $C$, its positivity is already well understood. Davenport [1959, §7] showed the sufficiency of a certain $p$-adic solubility property, invariant under equivalence (invertible change of basis). He also showed that any cubic form that is nondegenerate in at least ten variables has this property [Davenport 1959, Lemma 2.8]. If $C$ is degenerate, then it is equivalent to a cubic form $C^{*}$ in which $n_{1} \leqslant n-1$ variables appear explicitly so that $h\left(C^{*}\right) \leqslant n_{1}$ and we may repeat the argument. Since $h(C) \geqslant 25$, and since the $h$-invariant is invariant under equivalence, we conclude that $\mathfrak{S}>0$.

For positivity of the singular integral, we begin by establishing the equivalence of the definitions (1-6) and (1-8). Lemma 5.2 provides the appropriate analogy to [Schmidt 1982b, Lemma 11]. Thus, following Chapter 11 therein shows that the two definitions are equivalent.

We now work with the definition (1-8). We claim that $I_{L}(\boldsymbol{f}) \gg 1$. Since $w(\boldsymbol{x}) \gg 1$ for $x \in B:=\left\{x \in \mathbb{R}^{n}:|x| \leqslant \frac{1}{2}\right\}$, it suffices to show that

$$
\int_{B} \Psi_{L}(f(x)) \mathrm{d} \boldsymbol{x} \gg 1
$$

Define a real manifold

$$
\mathcal{M}=\left\{C=L_{1}=\cdots=L_{r}=0\right\} \subseteq \mathbb{R}^{n} .
$$

All of our forms have odd degree, so $\mathcal{M} \cap A \neq\{\boldsymbol{0}\}$ for every $(r+2)$-dimensional subspace $A$ of $\mathbb{R}^{n}$. Thus, by [Schmidt 1982a, Lemma 1], $\operatorname{dim}(\mathcal{M}) \geqslant n-r-1$. The argument of [Schmidt 1982b, Lemma 2] now confirms (7-1), thereby establishing the positivity of $\chi_{w}$. This completes the proof of Theorem 1.1.

\section{A more general result}

In this section, we prove Theorem 1.2. We begin by establishing that, if $\boldsymbol{\alpha} \in \mathbb{R}^{r} \backslash\{\mathbf{0}\}$, then $\boldsymbol{\alpha} \cdot \boldsymbol{L}$ is not a rational form. Suppose that $\boldsymbol{\alpha} \cdot \boldsymbol{L}$ is a rational form, for some $\boldsymbol{\alpha} \in \mathbb{R}^{r}$. Then $\Lambda \boldsymbol{\alpha}=\boldsymbol{q}$ for some $\boldsymbol{q} \in \mathbb{Q}^{n}$, where $\Lambda$ is given by (2-10). Note that $\Lambda$ has full rank since its entries are algebraically independent over $\mathbb{Q}$ and its $r \times r$ minors are nontrivial integer polynomials in these entries. It therefore follows from $\Lambda \boldsymbol{\alpha}=\boldsymbol{q}$ that $\alpha_{1}, \ldots, \alpha_{r}$ are rational functions in the entries of $\Lambda_{V}$ over $\mathbb{Q}$, where $V$ is as in Section 5 and $\Lambda_{V}$ is the submatrix of $\Lambda$ determined by taking rows indexed by $V$. Let $i \in\{1,2, \ldots, n\} \backslash V$, and consider the equation

$$
\alpha_{1} \lambda_{1, i}+\alpha_{r} \lambda_{r, i}=q_{i}
$$

Since $\alpha_{1}, \ldots, \alpha_{r}$ are rational functions in the entries of $\Lambda_{V}$ over $\mathbb{Q},(8-1)$ and the 
algebraic independence of the entries of $\Lambda$ necessitate that $\boldsymbol{\alpha}=\mathbf{0}$. We conclude that, if $\boldsymbol{\alpha} \in \mathbb{R}^{r} \backslash\{\boldsymbol{0}\}$, then $\boldsymbol{\alpha} \cdot \boldsymbol{L}$ is not a rational form.

By rescaling if necessary, we may assume that $C$ has integer coefficients. By Theorem 1.1, we may now assume that $h \leqslant 16+8 r$, and so $n-h>r$. Write

$$
C=A_{1} B_{1}+\cdots+A_{h} B_{h},
$$

where $A_{1}, \ldots, A_{h}$ are rational linear forms and $B_{1}, \ldots, B_{h}$ are rational quadratic forms. The vector space defined by

$$
A_{1}=\cdots=A_{h}=0
$$

has a rational subspace of dimension $n-h$, by the rank-nullity theorem. Let $z_{1}, \ldots, z_{n-h}$ be linearly independent integer points in this subspace. Define

$$
L_{i}^{\prime}(\boldsymbol{y})=L_{i}\left(y_{1} z_{1}+\cdots+y_{n-h} z_{n-h}\right) \quad(1 \leqslant i \leqslant r) .
$$

We seek to show that $\boldsymbol{L}^{\prime}\left(\mathbb{Z}^{n-h}\right)$ is dense in $\mathbb{R}^{r}$. Writing $z_{j}=\left(z_{j, 1}, \ldots, z_{j, n}\right)$ $(1 \leqslant j \leqslant n-h)$ and recalling (2-9),

$$
L_{i}^{\prime}(\boldsymbol{y})=\sum_{j \leqslant n-h} \lambda_{i, j}^{\prime} y_{j}
$$

where

$$
\lambda_{i, j}^{\prime}=\sum_{k \leqslant n} \lambda_{i, k} z_{j, k} \quad(1 \leqslant i \leqslant r, 1 \leqslant j \leqslant n-h) .
$$

Lemma 8.1. The $\lambda_{i, j}^{\prime}$ are algebraically independent over $\mathbb{Q}$.

Proof. Extend $z_{1}, \ldots, z_{n-h}$ to a basis $z_{1}, \ldots, z_{n}$ for $\mathbb{Q}^{n}$, and define

$$
\lambda_{i, j}^{\prime}=\sum_{k \leqslant n} \lambda_{i, k} z_{j, k} \quad(1 \leqslant i \leqslant r, n-h<j \leqslant n) .
$$

We now have an invertible rational matrix

$$
Z=\left(\begin{array}{ccc}
z_{1,1} & \cdots & z_{1, n} \\
\vdots & & \vdots \\
z_{n, 1} & \cdots & z_{n, n}
\end{array}\right),
$$

where $z_{j}=\left(z_{j, 1}, \ldots, z_{j, n}\right)(1 \leqslant j \leqslant n)$. Put

$$
\Lambda^{\prime}=\left(\begin{array}{ccc}
\lambda_{1,1}^{\prime} & \cdots & \lambda_{r, 1}^{\prime} \\
\vdots & & \vdots \\
\lambda_{1, n}^{\prime} & \cdots & \lambda_{r, n}^{\prime}
\end{array}\right)
$$

and note that $\Lambda^{\prime}=Z \Lambda$. 
We shall prove, a fortiori, that the entries of $\Lambda^{\prime}$ are algebraically independent over $\mathbb{Q}$. Let $P^{\prime}$ be a rational polynomial in $r n$ variables such that $P^{\prime}\left(\Lambda^{\prime}\right)=0$. Define a rational polynomial $P$ in $r n$ variables by

$$
P(\Xi)=P^{\prime}(Z \Xi), \quad \Xi \in \mathrm{Mat}_{n \times r} .
$$

Now

$$
P(\Lambda)=P^{\prime}(Z \Lambda)=P^{\prime}\left(\Lambda^{\prime}\right)=0,
$$

so the algebraic independence of the entries of $\Lambda$ forces $P$ to be the zero polynomial. Since

$$
P^{\prime}(\Xi)=P\left(Z^{-1} \Xi\right)
$$

identically, the polynomial $P^{\prime}$ must also be trivial.

Thus, the entries of the matrix

$$
A=\left(\begin{array}{ccc}
\lambda_{1,1}^{\prime} & \cdots & \lambda_{1, n-h}^{\prime} \\
\vdots & & \vdots \\
\lambda_{r, 1}^{\prime} & \cdots & \lambda_{r, n-h}^{\prime}
\end{array}\right)
$$

are algebraically independent over $\mathbb{Q}$, and we seek to show that

$$
\left\{A \boldsymbol{x}: \boldsymbol{x} \in \mathbb{Z}^{n-h}\right\}
$$

is dense in $\mathbb{R}^{r}$. We put $A$ in the form $\left(I \mid \Lambda^{\prime \prime}\right)$, where $I$ is the $r \times r$ identity matrix and $\Lambda^{\prime \prime}$ is an $r \times(n-h-r)$ matrix, by the following operations.

(i) Divide the top row by $A_{11}$ so that now $A_{11}=1$.

(ii) Subtract multiples of the top row from other rows so that

$$
A_{21}=\cdots=A_{r 1}=0 .
$$

(iii) Proceed similarly for columns $2,3, \ldots, r$.

It suffices to show that the image of $\mathbb{Z}^{n-h}$ under left multiplication by $\left(I \mid \Lambda^{\prime \prime}\right)$ is dense in $\mathbb{R}^{r}$.

Lemma 8.2. The entries of $\Lambda^{\prime \prime}$ are algebraically independent over $\mathbb{Q}$.

Proof. After step (i), the entries of $A$ other than the top-left entry are algebraically independent. Indeed, suppose

$$
P\left(\frac{A_{12}}{A_{11}}, \ldots, \frac{A_{1, n-h}}{A_{11}},\left(A_{i j}\right) \underset{\substack{2 \leqslant i \leqslant r \\ 1 \leqslant j \leqslant n-h}}{2}\right)=0
$$

for some polynomial $P$ with rational coefficients, where the $A_{i j}$ are the entries of $A$ prior to step (i). For some $t \in \mathbb{N}$, we can multiply the left-hand side by $A_{11}^{t}$ to

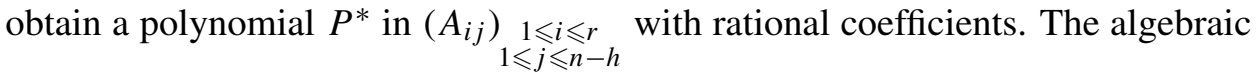


independence of the $A_{i j}$ implies that $P^{*}$ is the zero polynomial, and so $P$ must also be the zero polynomial.

After step (ii), the entries of $A$ excluding the first column are algebraically independent. Indeed, suppose

$$
P\left(A_{12}, \ldots, A_{1, n-h},\left(A_{i j}-A_{i 1} A_{1 j}\right) \underset{\substack{2 \leqslant i \leqslant r \\ 2 \leqslant j \leqslant n-h}}{2 \leqslant j}\right)=0
$$

for some polynomial $P$ with rational coefficients, where the $A_{i j}$ are the entries of $A$ prior to step (ii). The left-hand side may be regarded as a polynomial $P^{*}$ in the $A_{i j}$ $((i, j) \neq(1,1))$. The algebraic independence of the $A_{i j}((i, j) \neq(1,1))$ implies that $P^{*}$ is the zero polynomial, and so $P$ must also be the zero polynomial.

We may now ignore column 1 and deal with columns $2,3, \ldots, r$ similarly.

Next, consider the forms $L_{1}^{\prime \prime}, \ldots, L_{r}^{\prime \prime}$ given by

$$
L_{i}^{\prime \prime}(\boldsymbol{x})=\mu_{i, 1} x_{1}+\cdots+\mu_{i, n-h-r} x_{n-h-r} \quad(1 \leqslant i \leqslant r),
$$

where $\mu_{i, j}=\Lambda_{i j}^{\prime \prime}(1 \leqslant i \leqslant r, 1 \leqslant j \leqslant n-h-r)$. It remains to show that $\boldsymbol{L}^{\prime \prime}\left(\mathbb{Z}^{n-h-r}\right)$ is dense modulo 1 in $\mathbb{R}^{r}$. We shall in fact establish equidistribution modulo 1 of the values of $\boldsymbol{L}^{\prime \prime}\left(\mathbb{N}^{n-h-r}\right)$.

For this, we use a multidimensional Weyl criterion [Cassels 1957, p. 66]. With $m=n-h-r$, we need to show that, if $\boldsymbol{h} \in \mathbb{Z}^{r} \backslash\{\mathbf{0}\}$, then

$$
P^{-m} \sum_{x_{1}, \ldots, x_{m} \leqslant P} e\left(\boldsymbol{h} \cdot \boldsymbol{L}^{\prime \prime}(\boldsymbol{x})\right) \rightarrow 0
$$

as $P \rightarrow \infty$. The summation equals

$$
\prod_{j \leqslant m} \sum_{x_{j} \leqslant P} e\left(x_{j} \sum_{i \leqslant r} h_{i} \mu_{i, j}\right),
$$

so it suffices to show that

$$
\sum_{i \leqslant r} h_{i} \mu_{i, 1} \notin \mathbb{Q}
$$

This follows from the algebraic independence of the $\mu_{i, j}$, so we have completed the proof of Theorem 1.2.

\section{Equidistribution}

In this section, we prove Theorem 1.3. Let $\boldsymbol{k}$ be a fixed nonzero integer vector in $r$ variables. By a multidimensional Weyl criterion [Cassels 1957, p. 66], we need to show that

$$
N_{u}(P)^{-1} \sum_{\substack{|\boldsymbol{x}|<P \\ C(\boldsymbol{x})=0}} e(\boldsymbol{k} \cdot \boldsymbol{L}(\boldsymbol{x})) \rightarrow 0
$$


as $P \rightarrow \infty$, where

$$
N_{u}(P)=\#\left\{\boldsymbol{x} \in \mathbb{Z}^{n}:|\boldsymbol{x}|<P, C(\boldsymbol{x})=0\right\} .
$$

It is known that $P^{n-3} \ll N_{u}(P) \ll P^{n-3}$; see remark (B) in the introduction of [Schmidt 1985]. Thus, it remains to show that

$$
\sum_{\substack{|\boldsymbol{x}|<P \\ C(\boldsymbol{x})=0}} e(\boldsymbol{k} \cdot \boldsymbol{L}(\boldsymbol{x}))=o\left(P^{n-3}\right) .
$$

Let $\theta_{0}$ be a small positive real number, and let $U$ be as in (4-5). By rescaling if necessary, we may assume that $C$ has integer coefficients. By (2-7), the left-hand side of (9-1) is equal to

$$
\int_{U} S_{u}\left(\alpha_{0}, \boldsymbol{k}\right) \mathrm{d} \alpha_{0}
$$

where $S_{u}(\cdot, \cdot)$ is as defined in the introduction. Recall (2-9) and (2-10). Note that

$$
S_{u}\left(\alpha_{0}, \boldsymbol{k}\right)=g_{u}\left(\alpha_{0}, \lambda^{*}\right),
$$

where $g_{u}(\cdot, \cdot)$ is as defined in (2-12) and $\lambda^{*}=\Lambda \boldsymbol{k} \in \mathbb{R}^{n}$ is fixed.

For $q \in \mathbb{N}$ and $a \in \mathbb{Z}$, let $\mathfrak{N}^{\prime}(q, a)$ be the set of $\alpha_{0} \in U$ such that

$$
\left|q \alpha_{0}-a\right|<P^{2 \theta_{0}-3} \text {. }
$$

Recall that $C_{1}$ is a large positive real number. For positive integers $q \leqslant C_{1} P^{2 \theta_{0}}$, let $\mathfrak{N}^{\prime}(q)$ be the disjoint union of the sets $\mathfrak{N}^{\prime}(q, a)$ over integers $a$ that are relatively prime to $q$. Let $\mathfrak{N}^{\prime}$ be the disjoint union of the sets $\mathfrak{N}^{\prime}(q)$. By Lemma 2.1 and the classical pruning argument in [Davenport 2005, Lemma 15.1], it now suffices to prove that

$$
\int_{\mathfrak{N}^{\prime}} S_{u}\left(\alpha_{0}, \boldsymbol{k}\right) \mathrm{d} \alpha_{0}=o\left(P^{n-3}\right)
$$

Let $\alpha_{0} \in \mathfrak{N}^{\prime}(q, a)$, with $q \leqslant C_{1} P^{2 \theta_{0}}$ and $(a, q)=1$. Then

$$
S_{u}\left(\alpha_{0}, \boldsymbol{k}\right)=\sum_{\boldsymbol{y} \bmod q} e_{q}(a C(\boldsymbol{y})) S_{\boldsymbol{y}}\left(q, \beta_{0}, \lambda^{*}\right),
$$

where $\beta_{0}=\alpha_{0}-a / q$ and where in general we define

$$
S_{y}\left(q, \beta_{0}, \lambda\right)=\sum_{z:|\boldsymbol{y}+q z|<P} e\left(\beta_{0} C(\boldsymbol{y}+q z)+\lambda \cdot(\boldsymbol{y}+q z)\right) .
$$

Note that $\left|q \beta_{0}\right|<P^{2 \theta_{0}-3}$. Let $\mathfrak{Q}$ denote the set of positive integers $q \leqslant C_{1} P^{2 \theta_{0}}$ such that

$$
\left\|q \lambda_{v}^{*}\right\|<P^{7 \theta_{0}-1} \quad(1 \leqslant v \leqslant n)
$$


and put

$$
\mathfrak{Q}^{\prime}=\left\{q \in \mathbb{N}: q \leqslant C_{1} P^{2 \theta_{0}}\right\} \backslash \mathfrak{Q} .
$$

Suppose $q \in \mathfrak{Q}^{\prime}$, and let $j$ be such that $\left\|q \lambda_{j}^{*}\right\| \geqslant P^{7 \theta_{0}-1}$. To bound $S_{y}\left(q, \beta_{0}, \lambda^{*}\right)$, we reorder the summation, if necessary, so that the sum over $z_{j}$ is on the inside. We bound this inner sum using the Kusmin-Landau inequality [Graham and Kolesnik 1991, Theorem 2.1] and then bound the remaining sums trivially. Note that, as a function of $z_{j}$, the phase

$$
\beta_{0} C(\boldsymbol{y}+q z)+\lambda^{*} \cdot(\boldsymbol{y}+q z)
$$

has derivative

$$
\beta_{0} \frac{\partial}{\partial z_{j}} C(\boldsymbol{y}+q z)+q \lambda_{j}^{*}
$$

which is monotonic in at most two stretches. As $\left\|q \lambda_{j}^{*}\right\| \geqslant P^{7 \theta_{0}-1}$ and

$$
\beta_{0} \frac{\partial}{\partial z_{j}} C(\boldsymbol{y}+q z) \ll P^{2 \theta_{0}-1}
$$

over the range of summation, the Kusmin-Landau inequality tells us that the sum over $z_{j}$ is $O\left(P^{1-7 \theta_{0}}\right)$. The remaining sums are over ranges of length $O(P / q)$, so

$$
S_{y}\left(q, \beta_{0}, \lambda^{*}\right) \ll(P / q)^{n-1} P^{1-7 \theta_{0}} .
$$

Therefore,

$$
S_{u}\left(\alpha_{0}, \boldsymbol{k}\right) \ll q P^{n-7 \theta_{0}} .
$$

Since meas $\left(\mathfrak{N}^{\prime}(q)\right) \ll P^{2 \theta_{0}-3}$, we now have

$$
\sum_{q \in \mathfrak{Q}^{\prime}} \int_{\mathfrak{N}^{\prime}(q)} S_{u}\left(\alpha_{0}, \boldsymbol{k}\right) \mathrm{d} \alpha_{0} \ll \sum_{q \leqslant C_{1} P^{2 \theta_{0}}} P^{2 \theta_{0}-3} q P^{n-7 \theta_{0}}=o\left(P^{n-3}\right) .
$$

It therefore remains to show that

$$
\sum_{q \in \mathfrak{Q}} \int_{\mathfrak{N}^{\prime}(q)} S_{u}\left(\alpha_{0}, \boldsymbol{k}\right) \mathrm{d} \alpha_{0}=o\left(P^{n-3}\right) .
$$

We shall need to study the more general exponential sums $g_{u}\left(\alpha_{0}, \lambda\right)$. Let $q \in \mathbb{N}$ with $q \leqslant P$, and let $a, a_{1}, \ldots, a_{n} \in \mathbb{Z}$. Set $\alpha_{0}$ and $\lambda$ as in (3-1), and write

$$
g_{u}^{*}\left(\alpha_{0}, \lambda\right)=(P / q)^{n} S_{q, a, a} I_{u}\left(P^{3} \beta_{0}, P \boldsymbol{\beta}\right),
$$

where $S_{q, a, a}$ is given by (3-3) and where

Lemma 9.1. We have

$$
I_{u}\left(\gamma_{0}, \boldsymbol{\gamma}\right)=\int_{[-1,1]^{n}} e\left(\gamma_{0} C(\boldsymbol{x})+\boldsymbol{\gamma} \cdot \boldsymbol{x}\right) \mathrm{d} \boldsymbol{x} .
$$

$$
g_{u}\left(\alpha_{0}, \lambda\right)-g_{u}^{*}\left(\alpha_{0}, \lambda\right) \ll q P^{n-1}\left(1+P^{3}\left|\beta_{0}\right|+P|\boldsymbol{\beta}|\right) .
$$


Proof. First observe that

$$
g_{u}\left(\alpha_{0}, \lambda\right)=\sum_{\boldsymbol{y} \bmod q} e_{q}(a C(\boldsymbol{y})+\boldsymbol{a} \cdot \boldsymbol{y}) S_{\boldsymbol{y}}\left(q, \beta_{0}, \boldsymbol{\beta}\right)
$$

and that

$$
S_{\boldsymbol{y}}\left(q, \beta_{0}, \boldsymbol{\beta}\right)=\sum_{\substack{|\boldsymbol{x}|<P \\ \boldsymbol{x} \equiv \boldsymbol{y} \bmod q}} e\left(\beta_{0} C(\boldsymbol{x})+\boldsymbol{\beta} \cdot \boldsymbol{x}\right) .
$$

By [Browning 2009, Lemma 8.1], we now have

$$
\begin{aligned}
S_{\boldsymbol{y}}\left(q, \beta_{0}, \boldsymbol{\beta}\right) & =q^{-n} \int_{[-P, P]^{n}} e\left(\beta_{0} C(\boldsymbol{x})+\boldsymbol{\beta} \cdot \boldsymbol{x}\right) \mathrm{d} \boldsymbol{x}+O\left(\frac{P^{n-1}\left(1+P^{3}\left|\beta_{0}\right|+P|\boldsymbol{\beta}|\right)}{q^{n-1}}\right) \\
& =(P / q)^{n} I_{u}\left(P^{3} \beta_{0}, P \boldsymbol{\beta}\right)+O\left(\frac{P^{n-1}\left(1+P^{3}\left|\beta_{0}\right|+P|\boldsymbol{\beta}|\right)}{q^{n-1}}\right) .
\end{aligned}
$$

Substituting this into (9-3) yields (9-2).

Suppose $\alpha_{0} \in \mathfrak{N}^{\prime}(q, a)$ with $q \in \mathfrak{Q}$. With $\lambda=\lambda^{*}$ and $a_{v}$ the nearest integer to $q \lambda_{v}(1 \leqslant v \leqslant n)$, put (3-1) and $S_{u}^{*}\left(\alpha_{0}, \boldsymbol{k}\right)=g_{u}^{*}\left(\alpha_{0}, \lambda^{*}\right)$. In light of the inequalities

$$
1 \leqslant q \leqslant C_{1} P^{2 \theta_{0}}, \quad\left|q \beta_{0}\right|<P^{2 \theta_{0}-3}, \quad|q \boldsymbol{\beta}|<P^{7 \theta_{0}-1},
$$

the error bound (9-2) implies that

$$
S_{u}\left(\alpha_{0}, \boldsymbol{k}\right)-S_{u}^{*}\left(\alpha_{0}, \boldsymbol{k}\right) \ll P^{n-1+7 \theta_{0}} .
$$

Since

$$
\operatorname{meas}\left(\bigcup_{q \in \mathfrak{Q}} \mathfrak{N}^{\prime}(q)\right) \ll P^{4 \theta_{0}-3},
$$

it now suffices to prove that

$$
\sum_{q \in \mathfrak{Q}} \int_{\mathfrak{N}^{\prime}(q)} S_{u}^{*}\left(\alpha_{0}, \boldsymbol{k}\right) \mathrm{d} \alpha_{0}=o\left(P^{n-3}\right) .
$$

The final ingredient that we need for a satisfactory mean-value estimate is an unweighted analogue of (5-1).

Lemma 9.2. We have

$$
I_{u}\left(\gamma_{0}, \boldsymbol{\gamma}\right) \ll \frac{1}{1+\left|\gamma_{0}\right|^{h / 8-\varepsilon}+|\boldsymbol{\gamma}|^{1 / 3}} .
$$

Proof. Since $I_{u}\left(\gamma_{0}, \boldsymbol{\gamma}\right) \ll 1$, we may assume that $\left|\gamma_{0}\right|+|\boldsymbol{\gamma}|$ is large. Specializing $(q, a, \boldsymbol{a})=(1,0, \mathbf{0})$ in $(9-2)$, it follows that

$$
I_{u}\left(\gamma_{0}, \boldsymbol{\gamma}\right)=P^{-n} g_{u}\left(\gamma_{0} / P^{3}, \boldsymbol{\gamma} / P\right)+O\left(P^{-1}\left(\left|\gamma_{0}\right|+|\boldsymbol{\gamma}|\right)\right) .
$$


Since $I_{u}\left(\gamma_{0}, \gamma\right)$ is independent of $P$, we are free to choose $P=\left(\left|\gamma_{0}\right|+|\boldsymbol{\gamma}|\right)^{n}$. By Lemma 5.1, with $\xi=\varepsilon / n^{2}$, we now have

so

$$
I_{u}\left(\gamma_{0}, \boldsymbol{\gamma}\right) \ll P^{\varepsilon / n^{2}}\left|\gamma_{0}\right|^{-h / 8}+\frac{\left|\gamma_{0}\right|+|\boldsymbol{\gamma}|}{P} \ll P^{\varepsilon / n^{2}}\left|\gamma_{0}\right|^{-h / 8},
$$

$$
I_{u}\left(\gamma_{0}, \boldsymbol{\gamma}\right) \ll \frac{\left(\left|\gamma_{0}\right|+|\boldsymbol{\gamma}|\right)^{\varepsilon / n}}{\left|\gamma_{0}\right|^{h / 8}} .
$$

Let $C_{4}$ be a large positive constant. As $\left|\gamma_{0}\right|+|\gamma|$ is large and $h \geqslant 17$, the desired inequality follows from (9-6) if $|\boldsymbol{\gamma}|<C_{4}\left|\gamma_{0}\right|$. Thus, we may assume that $|\boldsymbol{\gamma}| \geqslant C_{4}\left|\gamma_{0}\right|$. Choose $j \in\{1,2, \ldots, n\}$ such that $|\boldsymbol{\gamma}|=\left|\gamma_{j}\right|$. Observe that

$$
I_{u}\left(\gamma_{0}, \boldsymbol{\gamma}\right) \ll \sup _{-1 \leqslant x_{i} \leqslant 1(i \neq j)}\left|\int_{-1}^{1} e\left(\gamma_{0} C(\boldsymbol{x})+\gamma_{j} x_{j}\right) \mathrm{d} x_{j}\right| .
$$

As $\left|\gamma_{j}\right| \geqslant C_{4}\left|\gamma_{0}\right|$, the bound in [Vaughan 1997, Theorem 7.3] now implies that

$$
I_{u}\left(\gamma_{0}, \boldsymbol{\gamma}\right) \ll\left|\gamma_{j}\right|^{-1 / 3}=|\boldsymbol{\gamma}|^{-1 / 3} .
$$

Combining this with (9-6) gives

$$
I_{u}\left(\gamma_{0}, \boldsymbol{\gamma}\right) \ll \frac{\left(\left|\gamma_{0}\right|+|\boldsymbol{\gamma}|\right)^{\varepsilon / n}}{\left|\gamma_{0}\right|^{h / 8}+|\boldsymbol{\gamma}|^{1 / 3}\left(\left|\gamma_{0}\right|+|\boldsymbol{\gamma}|\right)^{\varepsilon / n}} \ll \frac{|\boldsymbol{\gamma}|^{\varepsilon / n}}{\left|\gamma_{0}\right|^{h / 8}+|\boldsymbol{\gamma}|^{1 / 3+\varepsilon / n}} .
$$

Considering cases and recalling that $h \leqslant n$, we now have

$$
I_{u}\left(\gamma_{0}, \boldsymbol{\gamma}\right) \ll \frac{1}{\left|\gamma_{0}\right|^{h / 8-\varepsilon}+|\boldsymbol{\gamma}|^{1 / 3}} .
$$

This delivers the sought estimate (9-5) since $\left|\gamma_{0}\right|+|\boldsymbol{\gamma}| \gg 1$.

Let $\alpha_{0} \in \mathfrak{N}^{\prime}(q, a)$, with $q \in \mathfrak{Q}$ and $(a, q)=1$. The inequalities (3-6), (9-5), and $h \geqslant 17$ give

$$
S_{u}^{*}\left(\alpha_{0}, \boldsymbol{k}\right) \ll P^{n} q^{-2-\varepsilon}\left(1+P^{3}\left|\alpha_{0}-a / q\right|\right)^{-1-\varepsilon} F(\boldsymbol{k} ; q, P)^{\varepsilon},
$$

where

Therefore,

$$
F(\boldsymbol{k} ; q, P)=\prod_{v \leqslant n}\left(q+P\left\|q \lambda_{v}^{*}\right\|\right)^{-1}
$$

$$
\begin{aligned}
& \sum_{q \in \mathfrak{Q}} \int_{\mathfrak{N}^{\prime}(q)}\left|S_{u}^{*}\left(\alpha_{0}, \boldsymbol{k}\right)\right| F(\boldsymbol{k} ; q, P)^{-\varepsilon} \mathrm{d} \alpha_{0} \ll P^{n} \sum_{q \in \mathbb{N}} q^{-1-\varepsilon} \int_{\mathbb{R}}\left(1+P^{3}\left|\beta_{0}\right|\right)^{-1-\varepsilon} \mathrm{d} \beta_{0} \\
& \ll P^{n-3} .
\end{aligned}
$$

As $\boldsymbol{k}$ is a fixed nonzero vector, we have $1 \ll|\boldsymbol{k}| \ll 1$. In particular, by (6-2), we have $1 \ll|\Lambda \boldsymbol{k}| \ll 1$. Thus, Corollary 6.2 gives

$$
F(\boldsymbol{k} ; q, P) \leqslant \mathcal{F}(\boldsymbol{k} ; P)=o(1)
$$


as $P \rightarrow \infty$. Coupling this with (9-7) yields (9-4), completing the proof of Theorem 1.3.

\section{The singular locus}

The singular locus of $C$ is the complex variety cut out by vanishing of $\nabla C$. Let $\mathcal{S}$ be the singular locus of $C$, and let $\sigma$ be the affine dimension of $\mathcal{S}$. Let $h$ be the $h$-invariant of $C$, and let $A_{1}, \ldots, A_{h}, B_{1}, \ldots, B_{h}$ be as in (1-1). Then $\mathcal{S}$ contains the variety $\left\{A_{1}=\cdots=A_{h}=B_{1}=\cdots=B_{h}=0\right\}$, and so $\sigma \geqslant n-2 h$. In particular, the conclusions of Theorem 1.1 are valid if the hypothesis (1-2) is replaced by the condition

$$
n-\sigma>32+16 r
$$

Thus, these conclusions hold for any nonsingular cubic form in more than $32+16 r$ variables.

However, one could improve upon this using a direct approach. Note that $h$ could be replaced by $n-\sigma$ in Lemma 2.1; the resulting lemma would be almost identical to [Birch 1962, Lemma 4.3], and again the weights and lower-order terms are of no significance. The remainder of the analysis would be identical and lead us to conclude that Theorem 1.1 is valid with $h$ replaced by $n-\sigma$. We could even use the same argument for positivity of the singular series, for if $r \geqslant 1$, then

$$
h \geqslant \frac{n-\sigma}{2}>\frac{16+8 r}{2} \geqslant 12>9 .
$$

In particular, the conclusions of Theorem 1.1 would hold for any nonsingular cubic form in more than $16+8 r$ variables. Similarly, Theorem 1.3 is valid with $h$ replaced by $n-\sigma$, and so its conclusion would hold for any nonsingular cubic form in more than sixteen variables.

Finally, we challenge the reader to improve upon these statements using more sophisticated technology, for instance to reduce the number of variables needed to solve the system (1-7). It is likely that van der Corput differencing could be profitably incorporated, similarly to [Heath-Brown 2007]. One might also hope to do better by assuming that $C$ is nonsingular, as in [Heath-Brown 1983].

\section{Acknowledgments}

The author is very grateful towards Trevor Wooley for his energetic supervision. Many thanks are also owed to the anonymous referee for carefully reading this manuscript and offering helpful suggestions. 


\section{References}

[Bentkus and Götze 1999] V. Bentkus and F. Götze, "Lattice point problems and distribution of values of quadratic forms", Ann. of Math. (2) 150:3 (1999), 977-1027. MR 2001b:11087 Zbl 0979.11048

[Birch 1962] B. J. Birch, "Forms in many variables", Proc. Roy. Soc. (A) 265 (1962), 245-263. MR 27 \#132 Zbl 0103.03102

[Browning 2009] T. D. Browning, Quantitative arithmetic of projective varieties, Progress in Mathematics 277, Birkhäuser, Basel, 2009. MR 2010i:11004 Zbl 1188.14001

[Browning et al. 2015] T. D. Browning, R. Dietmann, and D. R. Heath-Brown, "Rational points on intersections of cubic and quadric hypersurfaces", J. Inst. Math. Jussieu 14:4 (2015), 703-749. MR 3394125 Zbl 1327.11043

[Cassels 1957] J. W. S. Cassels, An introduction to Diophantine approximation, Cambridge Tracts in Mathematics and Mathematical Physics 45, Cambridge University, New York, 1957. MR 19,396h Zbl 0077.04801

[Dani and Margulis 1993] S. G. Dani and G. A. Margulis, "Limit distributions of orbits of unipotent flows and values of quadratic forms", pp. 91-137 in I. M. Gelfand Seminar, edited by S. Gelfand and S. Gindikin, Advances in Soviet Mathematics 16, American Mathematical Society, Providence, RI, 1993. MR 95b:22024 Zbl 0814.22003

[Davenport 1959] H. Davenport, "Cubic forms in thirty-two variables", Philos. Trans. Roy. Soc. London. (A) 251:993 (1959), 193-232. MR 21 \#4136 Zbl 0084.27202

[Davenport 2005] H. Davenport, Analytic methods for Diophantine equations and Diophantine inequalities, 2nd ed., Cambridge University, 2005. MR 2006a:11129 Zbl 1125.11018

[Davenport and Heilbronn 1946] H. Davenport and H. Heilbronn, "On indefinite quadratic forms in five variables", J. London Math. Soc. 21:3 (1946), 185-193. MR 8,565e Zbl 0060.11914

[Davenport and Lewis 1964] H. Davenport and D. J. Lewis, "Non-homogeneous cubic equations", J. London Math. Soc. 39 (1964), 657-671. MR 29 \#4731 Zbl 0125.02402

[Eskin et al. 1998] A. Eskin, G. A. Margulis, and S. Mozes, "Upper bounds and asymptotics in a quantitative version of the Oppenheim conjecture", Ann. of Math. (2) 147:1 (1998), 93-141. MR 99a:11043 Zbl 0906.11035

[Freeman 2002] D. E. Freeman, "Asymptotic lower bounds and formulas for Diophantine inequalities", pp. 57-74 in Number theory for the millennium, II (Urbana, IL, 2000), edited by M. A. Bennett et al., A K Peters, Natick, MA, 2002. MR 2003j:11035 Zbl 1042.11022

[Graham and Kolesnik 1991] S. W. Graham and G. Kolesnik, Van der Corput's method of exponential sums, London Mathematical Society Lecture Note Series 126, Cambridge University, 1991. MR 92k:11082 Zbl 0713.11001

[Heath-Brown 1983] D. R. Heath-Brown, "Cubic forms in ten variables", Proc. London Math. Soc. (3) 47:2 (1983), 225-257. MR 85b:11025 Zbl 0494.10012

[Heath-Brown 1996] D. R. Heath-Brown, "A new form of the circle method, and its application to quadratic forms”, J. Reine Angew. Math. 481 (1996), 149-206. MR 97k:11139 Zbl 0857.11049

[Heath-Brown 2007] D. R. Heath-Brown, "Cubic forms in 14 variables", Invent. Math. 170:1 (2007), 199-230. MR 2010a:11063 Zbl 1135.11031

[Margulis 1989] G. A. Margulis, "Discrete subgroups and ergodic theory", pp. 377-398 in Number theory, trace formulas and discrete groups (Oslo, 1987), edited by K. E. Aubert et al., Academic Press, Boston, 1989. MR 90k:22013a Zbl 0675.10010

[Sargent 2014] O. Sargent, "Equidistribution of values of linear forms on quadratic surfaces", Algebra Number Theory 8:4 (2014), 895-932. MR 3248989 Zbl 1314.11018 
[Schmidt 1982a] W. M. Schmidt, "On cubic polynomials, IV: Systems of rational equations", Monatsh. Math. 93:4 (1982), 329-348. MR 83m:10063 Zbl 0481.10015

[Schmidt 1982b] W. M. Schmidt, "Simultaneous rational zeros of quadratic forms", pp. 281-307 in Séminaire de Théorie des Nombres: Séminaire Delange-Pisot-Poitou (Paris, 1980-1981), edited by M.-J. Bertin, Progress in Mathematics 22, Birkhäuser, Boston, 1982. MR 84g:10041 Zbl 0492.10017

[Schmidt 1985] W. M. Schmidt, "The density of integer points on homogeneous varieties", Acta Math. 154:3-4 (1985), 243-296. MR 86h:11027 Zbl 0561.10010

[Vaughan 1997] R. C. Vaughan, The Hardy-Littlewood method, 2nd ed., Cambridge Tracts in Mathematics 125, Cambridge University, 1997. MR 98a:11133 Zbl 0868.11046

[Wooley 2003] T. D. Wooley, “On Diophantine inequalities: Freeman's asymptotic formulae”, in Proceedings of the Session in Analytic Number Theory and Diophantine Equations (Bonn, 2002), edited by D. R. Heath-Brown and B. Z. Moroz, Bonner Mathematische Schriften 360, Universität Bonn, 2003. MR 2005d:11048 Zbl 1196.11055

Communicated by Roger Heath-Brown

Received 2015-04-29 Revised 2015-11-09 Accepted 2015-12-27

sam.chow@bristol.ac.uk

School of Mathematics, University of Bristol, University Walk, Bristol, BS8 1TW, United Kingdom 


\section{Algebra \& Number Theory}

msp.org/ant

\section{EDITORS}

MANAGING EDITOR

Bjorn Poonen

Massachusetts Institute of Technology

Cambridge, USA

\author{
EDITORIAL BOARD CHAIR \\ David Eisenbud \\ University of California \\ Berkeley, USA
}

BOARD OF EDITORS

Georgia Benkart

Dave Benson

Richard E. Borcherds

John H. Coates

J-L. Colliot-Thélène

Brian D. Conrad

Hélène Esnault

Hubert Flenner

Sergey Fomin

Edward Frenkel

Andrew Granville

Joseph Gubeladze

Roger Heath-Brown

Craig Huneke

Kiran S. Kedlaya

János Kollár

Yuri Manin

Philippe Michel
University of Wisconsin, Madison, USA

University of Aberdeen, Scotland

University of California, Berkeley, USA

University of Cambridge, UK

CNRS, Université Paris-Sud, France

Stanford University, USA

Freie Universität Berlin, Germany

Ruhr-Universität, Germany

University of Michigan, USA

University of California, Berkeley, USA

Université de Montréal, Canada

San Francisco State University, USA

Oxford University, UK

University of Virginia, USA

Univ. of California, San Diego, USA

Princeton University, USA

Northwestern University, USA

École Polytechnique Fédérale de Lausanne
Susan Montgomery

Shigefumi Mori

Raman Parimala

Jonathan Pila

Anand Pillay

Victor Reiner

Peter Sarnak

Joseph H. Silverman

Michael Singer

Vasudevan Srinivas

J. Toby Stafford

Ravi Vakil

Michel van den Bergh

Marie-France Vignéras

Kei-Ichi Watanabe

Efim Zelmanov

Shou-Wu Zhang
University of Southern California, USA

RIMS, Kyoto University, Japan

Emory University, USA

University of Oxford, UK

University of Notre Dame, USA

University of Minnesota, USA

Princeton University, USA

Brown University, USA

North Carolina State University, USA

Tata Inst. of Fund. Research, India

University of Michigan, USA

Stanford University, USA

Hasselt University, Belgium

Université Paris VII, France

Nihon University, Japan

University of California, San Diego, USA

Princeton University, USA

\section{PRODUCTION}

production@msp.org

Silvio Levy, Scientific Editor

See inside back cover or msp.org/ant for submission instructions.

The subscription price for 2016 is US $\$ 290 /$ year for the electronic version, and $\$ 485 /$ year ( $+\$ 55$, if shipping outside the US) for print and electronic. Subscriptions, requests for back issues and changes of subscribers address should be sent to MSP.

Algebra \& Number Theory (ISSN 1944-7833 electronic, 1937-0652 printed) at Mathematical Sciences Publishers, 798 Evans Hall \#3840, c/o University of California, Berkeley, CA 94720-3840 is published continuously online. Periodical rate postage paid at Berkeley, CA 94704, and additional mailing offices.

ANT peer review and production are managed by EditFLOW ${ }^{\circledR}$ from MSP.

\section{PUBLISHED BY}

- mathematical sciences publishers

nonprofit scientific publishing

http://msp.org/

(C) 2016 Mathematical Sciences Publishers 


\section{Algebra \& Number Theory}

Volume $10 \quad$ No. $2 \quad 2016$

Kummer theory for Drinfeld modules

RICHARD PINK

Parity and symmetry in intersection and ordinary cohomology

SHENGHAO SUN and WeIZHE ZHENG

DANIEL KRIZ

Squarefree polynomials and Möbius values in short intervals and arithmetic progressions JONATHAN P. KEATING and ZEEV RUDNICK

Equidistribution of values of linear forms on a cubic hypersurface SAM CHOW 